\title{
MOTION-DEFINED CONTOUR PROCESSING IN EARLY VISUAL CORTEX
}

\author{
by \\ Amol Gharat \\ Department of Psychology \\ McGill University \\ Montreal, Quebec, Canada
}

June 2011

A thesis submitted to McGill University in partial fulfillment of the requirements of the degree of Master of Science

(C) Amol Gharat, 2011 


\section{Abstract}

From our daily experience it is very clear that relative motion cues can contribute to correctly indentifying object boundaries and for perceiving depth. Motiondefined contours are not only generated by the motion of objects in a scene, but also by the movement of an observer's head and body. However the neural mechanism involved in detecting these contours is still unknown. To explore this mechanism, I extracellularly recorded responses of neurons in Area 18 of anesthetized and paralyzed cats while they were presented with visual stimuli. The goal of this study was to determine if neurons in Area 18 that have been previously shown to detect luminance, texture- and contrast-defined contours cueinvariantly could also detect motion-defined contours. Motion-defined contour stimuli were generated by modulating the velocity of high spatial frequency sinusoidal luminance gratings (carrier gratings) by a squarewave envelope. The carrier gratings used were outside the luminance passband of a neuron, such that presence of carrier alone within the receptive field of a neuron did not elicit a response. It was found that most of the neurons in Area 18 that responded to contrast-defined contours also respond to motion-defined contours. The orientation and direction selectivity of these neurons for motion-defined contours was similar to that of luminance gratings. A given neuron also exhibited similar selectivity for the spatial frequency of the carrier grating of contrast- and motiondefined contours. These results suggest that Area 18 is a common brain area where different second-order contours are detected in a form-cue invariant manner, through a common neural mechanism. 


\section{Résumé}

Au quotidien, il est clair que les indices locaux de mouvement relatif contribuent à l'identification des bords des objets et à la perception de la profondeur. Les contours définis par le mouvement ne sont pas seulement générés par le mouvement des objets dans une scène, mais également par le mouvement de la tête et du corps de l'observateur. Cependant, les mécanismes neuronaux impliqués dans la détection de ces contours restent toujours inconnus. Pour étudier ces mécanismes, j'ai effectué des enregistrements électrophysologiques extracellulaires dans l'aire 18 de chats anesthésiés et paralysés pendant que des stimuli visuels leurs étaient présentés. Le but de cette étude était de déterminer si les neurones de l'aire 18 pour lesquels il a été montré qu'ils détectent indifféremment les contours définis par la luminance, la texture ou le contraste peuvent aussi détecter les contours définis par le mouvement. Les stimuli de contours définis par le mouvement étaient générés en modulant la vitesse de réseaux de luminances sinusoïdaux de haute fréquence spatiale (réseau porteur) par une enveloppe en créneau. Les réseaux porteurs utilisés étaient au-delà de la bande passante de luminance des neurones de façon à ce que la présentation du réseau porteur seul dans le champ récepteur d'un neurone ne génère pas de réponse. Il a alors été observé que la plupart des neurones de l'aire 18 qui répondent aux contours définis par le contraste répondent également aux contours définis par le mouvement. Les sélectivités à l'orientation et à la direction des contours définis par le mouvement de ces neurones étaient similaires à celles des réseaux de luminance. Un neurone donné présentait également la même sélectivité à la fréquence spatiale du réseau porteur pour les contours définis par le contraste et le mouvement. Ces résultats suggèrent que l'aire 18 est une aire d'intégration où différents contours de 
second-ordre sont détectés par un mécanisme commun, indépendamment de la nature des indices locaux. 


\section{Acknowledgements}

I would like to thank Prof. Curtis Baker for giving me the opportunity to conduct this study in his lab and for his able guidance throughout the project in designing the study, data analysis and editing the manuscript. I would like to thank Guangxing Li for providing the software for curve-fitting and Plexon data file analysis. Also, I would like to thank Prof. Baker, Guangxing Li, Vargha Talebi and Lynda Domazet for assistance with the experiments. I would like to thank Alexandre Reynaud for his help with translation of my thesis abstract into French. My sincere thanks to Prof. Keith Franklin for his support during my study and for helpful comments on my thesis. My deepest thanks to my family and friends who are always there, quitely supporting me.

This work was supported by the Canadian Institutes of Health Research grant MA-9685 to CLB. 


\section{Table of Contents}

Abstract ................................................................................................................ii

Résumé ................................................................................................................ iii

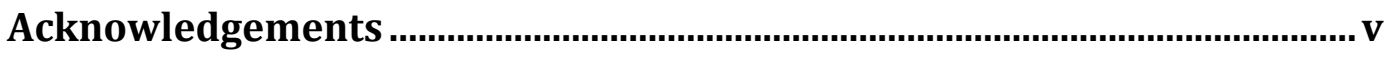

List of figures .......................................................................................... vii

Preface - Contribution of authors ........................................................... viii

Chapter 1 - Introduction .................................................................................

Chapter 2 - Materials and methods.............................................................. 7

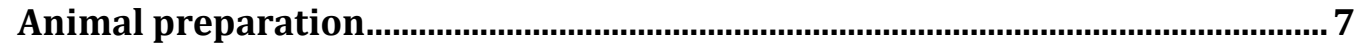

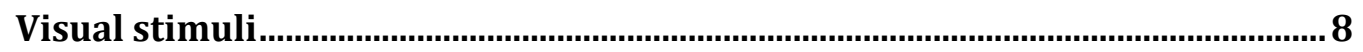

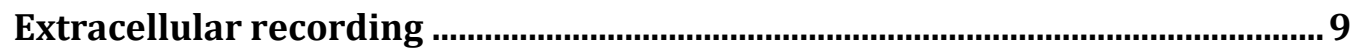

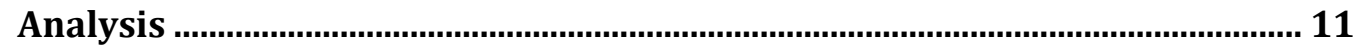

Chapter 3 - Results......................................................................................... 15

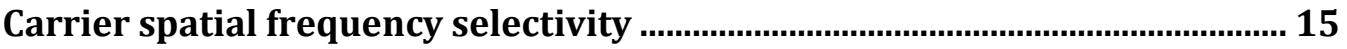

Envelope orientation selectivity ........................................................................ 16

Carrier temporal frequency tuning .................................................................... 18

Responsiveness to uni-directional and bi-directional gratings....................... 21

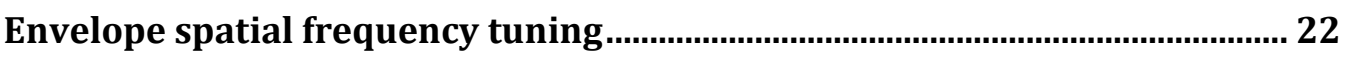

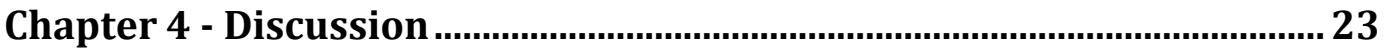

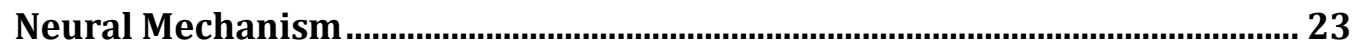

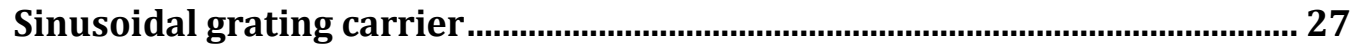

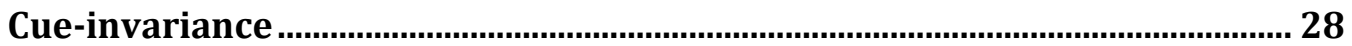

Chapter 5 - Summary \& Conclusion …......................................................... 31

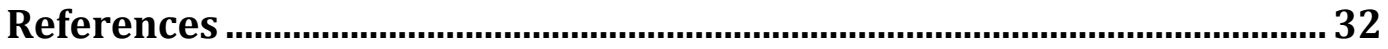




\section{List of figures}

Figure 1. Four types of grating stimuli used in this study.

Figure 2. Responses of a typical neuron to luminance modulation (LM), contrast modulation (CM) and velocity modulation (VM) gratings.

Figure 3. Relationship between optimal carrier spatial frequency for VM and CM gratings.

Figure 4. Orientation tuning of a typical neuron to LM gratings and envelope of VM and CM gratings.

Figure 5. Relationship between orientation selectivity for VM and LM gratings ........ 42

Figure 6. Carrier temporal frequency responses to "uni-directional" VM gratings ... 44

Figure 7. Carrier temporal frequency responses to "bi-directional" VM gratings ...... 46

Figure 8. Carrier temporal frequency responses to CM gratings. 48

Figure 9. Comparison of fall-off index for "uni-directional" VM gratings and CM gratings with LM gratings. 50

Figure 10. Comparison of peak response amplitudes to "uni-directional" and "bidirectional" VM gratings

Figure 11. Comparison of envelope spatial frequency (SF) tuning to illusory contours (IC), "uni-directional" and "bi-directional" VM gratings and spatial frequency tuning to luminance gratings...

Figure 12. A schematic model accounting for neuronal responses to luminance modulation (LM), contrast modulation (CM) and velocity modulation (VM) gratings. 


\section{Preface - Contribution of authors}

This thesis is written in manuscript form as permitted by the McGill University. It is composed of one manuscript, as listed below, with contribution of each author.

Gharat A and Baker CL Jr (2011) Motion-defined contour processing in early visual cortex. (To be submitted)

This study was designed by both Amol Gharat and Curtis Baker. In this study, animal preparation for the recording experiments was done by Amol Gharat and Curtis Baker, with assistance from two lab members - Guangxing and Vargha. The software for visual stimuli presentation and data acquisition was primarily built by Curtis Baker and it was further modified by Amol Gharat to generate the motion-defined boundary stimuli. Most of the data was collected by Amol Gharat except for a few recordings by Curtis Baker. All the data analysis and manuscript writing was performed by Amol Gharat with guidance from Curtis Baker. 


\section{Chapter 1 - Introduction}

Natural scenes abundantly contain local variations in luminance that facilitate figure-ground segregation. But, these first-order cues often introduce ambiguities and make figure-ground segregation a difficult task (Marr, 1982). For example, shadows introduce false luminance boundaries that do not correspond to objects' boundaries in a visual scene. However, our visual system is able to distinguish these false boundaries from real ones using other cues, including second-order information such as texture, contrast, color, or motion differences between object and its background. Particularly, relative motion between object and background is a powerful cue that can break camouflage when object and background have similar luminance, color, and texture. It can be sufficient to support perception of shape and size of three dimensional surfaces, and for depth ordering (Rogers \& Graham, 1979; Regan, 1989; Regan \& Hamstra 1992). This cue arises from motion parallax generated by an observer's movement, or from exogenous movement of objects in a scene.

Even though psychophysical studies have shown the importance of relative motion cues in figure-ground segregation, the neural mechanism to detect these motion-defined boundaries is still unknown. Single-unit recording experiments by Hubel and Wiesel (1962) on cats showed that orientation selectivity for luminance edges first originates in brain areas as early as primary visual cortex (V1). Simple cells in V1 have receptive fields with elongated excitatory and inhibitory areas lying adjacent and parallel to each other, which act as linear filters that perform linear summation of light intensity in their receptive 
fields. Hubel and Wiesel proposed a model in which receptive fields of simple cells are constructed by excitatory and inhibitory inputs from LGN cells arranged in alternating columns. A similar question could be asked in the case of motiondefined boundaries i.e. where does orientation selectivity for these boundaries originate and what is the neural mechanism behind it?

Several single-unit studies have tried to locate the brain areas responsive to motion-defined boundaries and understand the neural mechanism to detect these boundaries. Using temporal texture bars (dynamic random dot patterns moving on a stationary random dot background), (Albright 1992) reported that most of the neurons in area MT of macaque monkeys were selective for orientation of these bars and (Chaudhuri et al. 1997) found more than half of the neurons in area V1 of macaque monkeys selective for orientation of these bars. (Marcar et al. 2000) found a small fraction of neurons in macaque Area V1 and V2 that were selective for orientations of motion-defined boundaries. In macaque Area V4 (Mysore et al. $2004)$ reported a sizeable fraction of neurons (10-20\%) that were selective for kinetic patterns. Both these studies (Marcar et al. 2000) and (Mysore et al. 2004) used moving random dot texture patterns to generate motion-defined boundaries and they held these boundaries stationary in the receptive field of a neuron. (Zeki et al 2003) found a majority of neurons in macaque Area V3 and V3A selective to orientation of motion-defined bars made of random dot texture patterns. These studies suggest that motion-defined boundary selective neurons are present in different visual areas like V1, V2, V3, V3A, V4, and MT with higher percentage of them being in higher extrastriate areas. Although there is some discrepancy of 
results in early visual areas between different studies, it could be attributed to differences in stimuli used by them (Chaudhuri et al 1997; Marcar et al 2000). However there is a potential problem with this random dot texture patterns used in all these studies because these textures contained a broad range of spatial frequencies. Hence these texture patterns will contain energy within the luminance passband of a neuron, and therefore the response of a neuron could be due to local luminance (first-order) signals and not motion difference (second-order) cues. Such luminance signals or artifacts can be avoided by using a sinusoidal grating as a texture pattern with the spatial frequency higher than the neuron's luminance resolution.

Neuronal responses to contrast-defined (second-order) boundaries have been extensively studied in cat Area 18 (homolog to monkey V2) using singleunit recordings (Zhou \& Baker, 1993, 1996; Mareschal \& Baker, 1998a, 1998a, 1999). Contrast-defined boundaries used in these studies were constructed by a coarse spatial scale contrast modulation (envelope) of high spatial frequency sinusoidal grating (carrier) (Fig.1 B). Around half of the neurons in Area 18 responded to contrast-defined boundaries in a form-cue invariant manner i.e. they were tuned to the same orientation and motion direction of luminance (Fig. 1 A) (first-order) and contrast-defined (second-order) boundaries. In these studies carrier spatial frequency was constrained to lie outside a neuron's spatial frequency passband (measured using luminance grating) to ensure that the response of a neuron was genuinely second order and not due to first-order luminance signals. Surprisingly, these neurons showed narrow band-pass tuning 
for carrier spatial frequency. (Song \& Baker 2007) subsequently showed that these contrast-defined boundary responsive neurons also respond to texturedefined boundaries (second-order) and again in a form-cue invariant manner. Texture-defined boundaries (illusory contours), similar to contrast-defined boundaries were constructed using high spatial frequency sinusoidal gratings as a carrier, whose phase was modulated by square wave envelope. Neurons showed narrow band-pass tuning for carrier spatial frequency of texture-defined boundaries and were selective for similar carrier spatial frequencies. These results suggest that these neurons would be functionally useful in mediating responses to boundaries regardless of the cue that defines them, and this cue-invariance to different second-order boundaries might arise from a common nonlinear neuronal mechanism.

First and second order information are thought to be processed by two parallel pathways and this idea is supported by both psychophysical and neurophysiological experiments (Ledgeway \& Smith, 1994; Mather \& West, 1993a; Nishida et al., 1997; Scott-Samuel \& Georgeson, 1999; Baker \& Mareschal, 2001). First-order information can be detected by neurons acting as quasi-linear spatio-temporal filters. For detecting second-order information, neurophysiology experiments support a two-stage filter-rectify-filter (FRF) model (Mareshcal \& Baker, 1999), involving early filtering which is selective for local texture characteristics, followed by rectification, and the second-stage coarse scale spatio-temporal direction selective filtering (Wilson, 1999). The second stage filter has similar properties as the first-order filter, but it pools across a coarser 
spatial scale. Then these two parallel pathways converge onto a single neuron. This model is supported by recent neuroimaging (Zhan \& Baker, 2006) and single-unit neurophysiology (Song \& Baker, 2007) in Area 18 of cats.

In this study we hypothesize that these second-order responsive neurons in Area 18 of cats might also respond selectively to motion-defined boundaries, and that they might do so in a form-cue invariant manner. We used high spatial frequency sinusoidal gratings as texture (carrier) patterns, and relative motion between these textures to create motion-defined boundaries (Fig.1 C \& D). The spatial frequency of carrier gratings was optimized for each neuron such that it was well outside of the neuron's conventional luminance grating resolution in order to avoid luminance artifacts, i.e. motion of the carrier grating alone in the neuron's receptive field did not elicit any response.

A common motion-defined boundary occurs when an object exogenously moves in the visual field. In this case the retinal image of the background is nearly stationary, but the image of the object moves - we mimic this situation with a square wave envelope in which alternate half cycles contain either a moving or a stationary texture (carrier) (Fig. 1C) - a "uni-directional" motion boundary. We mimic another kind of motion boundary generated from motion parallax with a stimulus in which texture in alternate half cycle of the envelope moves in opposite directions (Fig.1D) - a "bi-directional" motion boundary. We restricted this study to shear motion boundaries, in which local motions are parallel to the edge and did not include compressive motion boundaries, in which local motions are perpendicular to the edge in order to avoid complexities of accretion deletion cue 
(Sary et al., 1994). We compared neurons' responses to motion-defined boundaries with contrast-defined and luminance-defined boundaries in order to check for form-cue invariance, and similarity between neural mechanism detecting contrast-defined and motion-defined boundaries.

We found that all contrast-defined boundary-responsive neurons also respond to "uni-directional" motion boundaries in a form-cue invariant manner, and with similar carrier spatial frequency tuning. Some but not all contrastdefined boundary-responsive neurons respond to "bi-directional" motion boundaries, typically with weaker responses than to "uni-directional" boundaries. Responses of these neurons' fit well with the FRF model indicating that motiondefined boundaries are processed by the same non-linear neural mechanism that processes contrast-defined boundaries. 


\section{Chapter 2 - Materials and methods}

\section{Animal preparation}

All experimental procedures were reviewed and approved by Animal Care Committee of McGill University. Cats were anesthetized using isoflurane/oxygen, and once the animal was asleep it was transferred to a surgery table where anesthesia was maintained using isoflurane delivered through a face mask. Methylcellulose gel was applied to both eyes to protect the corneas and a rectal thermistor was inserted to monitor temperature during surgery. Intervenous cannulation was performed on both hind legs, glycopyrrolate $(0.005 \mathrm{mg} / \mathrm{kg})$ and dexamethasone $(0.6 \mathrm{mg})$ were delivered inter-muscularly. The isoflurane face mask was removed and a loading dose of propofol $(5 \mathrm{mg} / \mathrm{kg})$ was delivered, with propofol then maintained at $6 \mathrm{mg} / \mathrm{kg} / \mathrm{hr}$ for subsequent surgery. EKG leads were connected to three limbs in order to monitor heart rate. Tracheal intubation was performed to provide a secure airway and the animal was secured on a stereotaxic apparatus. A respirator (ugo basile) was connected to deliver a mixture of $\mathrm{O} 2 / \mathrm{N} 2 \mathrm{O}(30: 70)$. End-tidal CO2 was monitored with a capnometer and maintained between 28 to $36 \mathrm{mmHg}$ by adjusting the respirator stroke volume and rate was fixed at 20 strokes/min. The sensor of a pulse-oximeter (Nonin) was placed into mouth, in contact with the tongue, to measure blood oxygen. Eye drops (atrophine and phenylephrine) were applied to the eyes, and neutral contact lenses were inserted. A craniotomy was made at Horseley-Clarke A3/L4, approximately $4 \times 4 \mathrm{~mm}$ to expose Area 18 . A duratomy was also performed when recording with multi-electrodes. EEG screws were inserted into the skull, away 
from the craniotomy. The craniotomy was covered with $2 \%$ agarose, and then with petroleum jelly. All surgical wounds were infused with bupivacaine, a local long-lasting anesthetic and the animal was wrapped in a heating pad to maintain temperature at $37.5^{\circ} \mathrm{C}$. The animal was paralyzed with a bolus injection of gallemine triethiodide (10-15 mg approx), and then paralysis was maintained during recording with an infusion rate of $10 \mathrm{mg} / \mathrm{kg} / \mathrm{hr}$. Fentanyl $(14 \mathrm{ug} / \mathrm{ml})$ and propofol $(10 \mathrm{mg} / \mathrm{ml})$ were also infused at a rate of $0.53 \mathrm{ml} / \mathrm{kg} / \mathrm{hr}$. Artificial pupils were placed in front of the eyes, and a flashlight was used to align them, by centering the reflected pinpoint of light. Appropriate spectacle lenses were selected using a slit retinoscope, and were placed in front of the eyes to provide refraction at a viewing distance of $57 \mathrm{~cm}$. An image of the retinal blood vessels and optic disk was back-projected on a tangent screen, and used to estimate the location of the area centralis of each eye.

\section{Visual stimuli}

Visual stimuli were generated by a Macintosh (Intel 4x2.66 GHz, 6GB) using Matlab with Psychophysics Toolbox (Brainard, 1997; Pelli, 1997) and presented on 17-inch CRT monitor (resolution 640x480 pixels, 75Hz). The CRT monitor was gamma corrected, as the carrier contrast used was very high $(70 \%$ contrast). The stimuli were confined within $480 \times 480$ pixels corresponding to 30x30 deg at a viewing distance of $57 \mathrm{~cm}$. Conventional luminance modulation (LM) sine wave gratings (Fig. 1 A) with contrast of $30 \%$ were used to measure luminance-passband of a neuron (spatial frequency, orientation and temporal frequency tuning). Contrast modulation (CM) gratings (Fig. 1B) were constructed 
by modulation of contrast of a carrier (texture pattern) by a low spatial frequency grating ("envelope"). The envelope of the contrast modulation grating was drifted with a temporal frequency lower than the neuron's optimum for LM gratings (Mareschal \& Baker, 1999). A high spatial frequency sinusoidal grating was used as a carrier, with a contrast of $70 \%$. Motion-defined boundaries were generated using "velocity modulation" (VM) gratings in which alternate half cycles of the envelope contained a texture (carrier) moving with different velocities. This boundary was parallel to the motion direction of the carrier (shear motion boundary). In particular, we tested two types of velocity modulation gratings, viz. "uni-directional" (Fig. 1 C) and "bi-directional" (Fig. 1 D). In "uni-directional" VM gratings, alternate half cycles of envelope contained moving or stationary carrier. "Bi-directional" boundaries were created by oppositely moving carriers. For CM gratings, the envelope was sinusoidal, while for VM gratings it was a square-wave. All stimuli were presented within a raised cosine-tapered, circular window against a gray background of the same luminance.

\section{Extracellular recording}

Spikes from single neurons were recorded extracellularly in Area 18 of cats with glass-coated Platinum/Iridium and parylene-coated tungsten single channel microelectrodes (Frederick Haer), and 16 channel multielectrodes (Neuronexus). Spikes were collected using a lab interface (Instrutech, ITC-18) with $100 \mu$ s resolution, and simultaneously the raw data signals were also acquired with a Plexon Recorder (filtered $5 \mathrm{~Hz}$ to $6 \mathrm{kHz}$, sampled at $40 \mathrm{~Hz}$ ) and streamed to hard disk for later analysis. Single-units were isolated using a window 
discriminator (Frederick Haer) and displayed on a delay-triggered digital oscilloscope. When recording with multielectrodes, spikes from one selected channel were analyzed online and used to guide the recording protocol mentioned below. A photocell was used for temporal registration of stimulus onset/offset timing and spike recordings.

Once spikes from a single neuron were isolated, a bar-shaped stimulus produced by a hand projector were used to probe the neuron's receptive field. Location of the receptive field, ocular dominance, eccentricity and approximate optimal orientation were measured. Then the CRT monitor was centered on the neuron's receptive field, and the non-dominant eye was occluded. Drifting sinewave luminance gratings were used to measure the neuron's luminance passband (spatial frequency, orientation and temporal frequency tuning). Each stimulus condition was randomly interleaved and repeatedly presented for 10-20 times to get the tuning curves. Then the neuron's optimal LM grating was presented in small circular patches in different locations on the screen to more accurately map the receptive field, and the screen was re-centered if necessary. To measure the size of the receptive field and check for surround suppression, the optimal LM grating was presented in circular patches of varying sizes centered on the receptive field.

Once parameters to LM gratings were optimized, responses to drifting contrast modulation $(\mathrm{CM})$ gratings (stationary carrier) were recorded, with an envelope orientation at the neuron's optimal luminance orientation and envelope spatial frequency equal to or lower than the neuron's optimal luminance spatial 
frequency (Mareschal \& Baker, 1999). A series of carrier spatial frequencies were tested, ranging from the neuron's luminance passband to values near the screen resolution, to find an optimal carrier spatial frequency. We classified a neuron as second-order responsive if it gave significant responses compared to spontaneous activity (t-test) at relatively high carrier -spatial frequencies that were well outside the luminance passband of the neuron, and if this spatial frequency tuning was band-pass. This condition of band-pass tuning was included to make sure that the neuron's response was genuinely second-order and not due to a nonlinearity in the screen which might give rise to a luminance signal at the envelope spatial frequency. If the neuron was classified as second-order responsive, then responses to velocity modulation (VM) gratings were recorded by testing series carrier spatial frequencies, with envelope orientation fixed to the neuron's optimal luminance orientation. If a neuron responded significantly to VM gratings, then envelope orientation tuning was measured using the neuron's optimal carrier spatial frequency with carrier orientation always kept perpendicular to the envelope orientation. The temporal frequency of the drifting carriers in velocity modulation gratings was varied, to study carrier temporal tuning properties. The temporal frequency response for the carrier of contrast modulation gratings was also obtained to compare with the one from velocity modulation gratings.

\section{Analysis}

Neurons were classified as either simple or complex cells by measuring the ratio of modulation to mean responses (AC/DC) to the neuron's optimal LM grating. If the ratio was greater than one, the neuron was classified as a simple 
type cell, otherwise it was classified as a complex cell (Skottun et al.,1991).

Neuronal responses used in the formulae below had spontaneous activity subtracted from them.

Spatial frequency tuning curves were fit with a gaussian function (DeAngelis et. al. 1994) to obtain an estimated optimal spatial frequency,

$$
R(s f)=k e^{-\left(s f-S F_{o p t} / \alpha\right)^{2}}+R_{o}
$$

where $\mathrm{k}, S F_{\text {opt }}, \alpha, R_{o}$ are free parameters and $R$ represents neuronal response at spatial frequency $s f$. A bootstrap resampling method (Efron \& Tibshirani, 1993) was used to estimate $95 \%$ confidence intervals for the obtained optimal spatial frequency $\left(S F_{\text {opt }}\right)$ value.

For orientation tuning curves, circular variance $(\mathrm{CV})$ was calculated as an index of tuning bandwidth (Marida 1972).

$$
C V=1-\frac{\left|\sum_{k} R_{k} \exp \left(i 2 \theta_{k}\right)\right|}{\sum_{k} R_{k}}
$$

where $R_{k}$ represents neuronal response at orientation $\theta_{k}$. Circular variance ranges from zero (sharp tuning) to unity (isotropic tuning). Optimal orientation was estimated as:

$$
O r i_{\text {opt }}=\arg \left(\frac{\sum_{k} R_{k} \exp \left(i 2 \theta_{k}\right)}{\sum_{k} R_{k}}\right)
$$

where arg denotes angular component of a complex number.

Motion direction selectivity of a neuron was measured by a direction selectivity index (DSI), 
$D S I=\left(R_{P}-R_{N}\right) /\left(R_{P}+R_{N}\right) \times 100 \%$

where $R_{P}$ is response of the neuron to its preferred direction of motion and $R_{N}$ is response to its non-preferred direction. DSI ranges from $0 \%$ (non-directional) to $100 \%$ (completely directional).

Symmetry of a neuron's responses to opposite directions of carrier motion for "uni-directional" VM and CM gratings was measured by a symmetry index (SI),

$S I=1-\frac{\sum_{k}\left(R_{k}-R_{-k}\right)^{2}}{\sum_{k}\left(R_{k}+R_{-k}\right)^{2}}$

where $R_{k}$ is response of the neuron to $\mathrm{VM}$ or $\mathrm{CM}$ gratings with carrier drifting at 'k' $\mathrm{Hz}$ and $R_{-k}$ is response to stimuli with carrier drifting at 'k' $\mathrm{Hz}$ in the opposite direction. SI would be 0 if the neuron responds only to one direction of carrier motion and not to the other (direction selective), and it would be 1 if the neuron responds equally to both directions of carrier motion (non-direction selective).

Proportional fall in response of a neuron at high temporal frequency compared to its optimal response is given by a fall-off index (FI), $F I=\left(R_{H}\right) /\left(R_{\max }\right)$

Fall-off index was calculated for responses of a neuron to LM gratings as well as VM and CM gratings. In the case of LM gratings, $R_{H}$ is response of the neuron to LM gratings drifting at $16 \mathrm{~Hz}$ and $R_{\max }$ is response to LM gratings at the optimal temporal frequency. In the case of VM and CM gratings, $R_{H}$ is response of the neuron to $\mathrm{VM}$ or $\mathrm{CM}$ gratings with carrier drifting at $16 \mathrm{~Hz}$ and $R_{\max }$ is response to the same grating at its optimal carrier temporal frequency. FI ranges from 0 
(response falls to spontaneous at $16 \mathrm{~Hz}$ ) to 1 (optimal response at16 Hz over the measured range of $0-16 \mathrm{~Hz}$ ).

Pearson's correlation coefficient was calculated for scatter plots comparing optimal carrier spatial frequencies for CM \& VM gratings and optimal orientations for LM gratings and envelope of VM gratings. Also, nonparametric, paired comparisons (Wilcoxon signed rank test) were used to evaluate whether a neuron exhibited similar preference for two kinds of gratings.

For recordings with multi-electrodes, spikes from only one channel were analyzed online to construct tuning curves. In later offline analysis, spikes from other channels were detected and classified using the Plexon Offline Sorter software. Isolated neurons from these channels were included in further analysis only if they showed very similar tuning to orientation and spatial frequency of LM gratings compared to the neuron recorded online. In some cases for recordings with single channel electrodes, offline sorting of spikes was performed to correct misclassifications by the window discriminator, and to isolate and assess lower amplitude spikes. 


\section{Chapter 3 - Results}

For this study, I recorded from 115 Area 18 neurons in 13 cats. Out of these, $64(55 \%)$ were classified as second order responsive neurons, as they responded significantly to contrast modulation (CM) gratings and showed bandpass tuning to its carrier spatial frequency. These second order responsive neurons were further tested with velocity modulation (VM) gratings (motion-defined) of two types, "uni-directional" and "bi-directional". Carrier spatial frequency and envelope orientation tuning were measured using "uni-directional" VM gratings, which gave stronger responses than "bi-directional" VM gratings. Note that it was not possible to measure selectivity for each second-order neuron that I recorded to all the stimuli parameters studied because of inability to hold stable recording for longer durations.

\section{Carrier spatial frequency selectivity}

Previous studies on second-order responsive neurons in Area 18 have shown that these neurons show narrow band-pass tuning to carrier spatial frequency of CM gratings well outside luminance passband (Zhou \& Baker,1993,1996; Mareschal \& Baker,1998; Tanaka \& Ohzawa,2006). Thus we wanted to see if these neurons would also show similar selectivity to carrier spatial frequency of VM gratings. The neuron in Fig.2 showed bandpass tuning to luminance gratings $(0.02-0.2 \mathrm{cpd}$, with a peak response at $0.08 \mathrm{cpd})$. The $\mathrm{CM}$ gratings were tested with varying carrier spatial frequencies, with envelope orientation fixed at the neuron's optimal luminance orientation. This neuron showed band-pass tuning to carrier spatial frequency and the response peaked at 
approximately $0.8 \mathrm{cpd}$. At this high spatial frequency range, the carrier signals were well beyond the neuron's luminance resolution, and hence it was classified as second-order responsive neuron. Then, this neuron was further tested with VM gratings for varying carrier spatial frequencies, with envelope orientation fixed at the neuron's optimal luminance orientation. This neuron showed similar bandpass tuning to carrier spatial frequency as shown for $\mathrm{CM}$ gratings and the response peaked at approximately $0.8 \mathrm{cpd}$.

The scatter plot in Fig. 3A shows a given neuron's optimal carrier spatial frequency for VM gratings against $\mathrm{CM}$ gratings for 30 neurons (9 simple and 21 complex). Points on the scatter plot are highly correlated $(\mathrm{r}(28)=0.7, \mathrm{p}<0.0001)$ and most of the points on the scatter plot lie close to the equality line, indicating that carrier spatial frequency tuning is similar for both types of gratings. Only 1 neuron out of 30 neurons had significantly different optimal carrier spatial frequencies for two stimuli (bootstrap method, C.I. $=95 \%$ ). The histogram plotted for differences in optimal carrier spatial frequencies between CM and VM gratings is centered on zero and mean of these differences is $0.15 \mathrm{cpd}$. This mean is small compared to the mean spatial frequency tuning bandwidths for CM (BW $=0.47 \mathrm{cpd})$ and $\mathrm{VM}(\mathrm{BW}=0.62 \mathrm{cpd})$.

\section{Envelope orientation selectivity}

In order to asses "form-cue invariance" for luminance- and motion-defined boundaries, like that previously demonstrated for luminance- and contrast-defined boundaries (Mareschal \& Baker, 1998a) we measured orientation selectivity of neurons to the envelope of "uni-directional" VM gratings, for comparison with 
that of LM gratings. Fig. 4A shows responses of a typical neuron that was tuned to orientation as well as motion direction of luminance gratings $\left(\right.$ Ori $i_{\text {opt }}=97 \mathrm{deg}$, DSI $=0.94)$. This neuron also showed similar orientation tuning for the envelope of VM gratings (Fig. 4B) (Ori $\left.i_{\text {opt }}=91 \mathrm{deg}\right)$, and was also direction-selective though to a somewhat smaller degree $(\mathrm{DSI}=0.55)$. For this particular neuron we also measured envelope orientation tuning for $\mathrm{CM}$ gratings (Fig. 4C) $\left(\right.$ Ori $i_{\text {opt }}=$ 96.1 , DSI $=0.37 \mathrm{deg}$ ), its tuning was also very similar to that for VM gratings. The scatter plot in Fig. 5A shows a given neuron's optimal envelope orientation for VM gratings against optimal orientation for LM gratings for 26 neurons (10 simple and 16 complex). Points on the scatter plot are highly correlated $(\mathrm{r}(24)=0.95, \mathrm{p}<0.0001)$ and most of the points lie close to the equality line, $58 \%(15 / 26)$ of the neurons had optimal orientation differences less than 15 degrees, and a maximum orientation difference of 37 degrees. There is a no significant difference between a given neuron's optimal orientation for LM gratings and optimal envelope orientation for VM gratings (Wilcoxon signed rank test, $\mathrm{p}=0.34)$. The histogram in Fig. $5 \mathrm{~B}$ shows that differences in optimal orientations are very small (mean $=-4.4 \mathrm{deg}$ ). The scatter plot in Fig. $5 \mathrm{C}$ shows a given neuron's envelope orientation circular variance (CV) for VM gratings against orientation circular variance (CV) for LM gratings. Most of the points (23 out of 26) lie above the equality line, suggesting that neuron's had broader tuning for envelope orientation of VM gratings compared to orientation tuning for LM gratings and $\mathrm{CV}$ for $\mathrm{VM}$ gratings is significantly greater than $\mathrm{CV}$ for $\mathrm{LM}$ gratings (Wilcoxon signed rank one-tailed test, $\mathrm{p}<0.05$ ). The scatter plot in Fig. 5D shows 
a given neuron's direction selectivity index for motion direction of envelope of VM gratings vs. motion direction of LM gratings. Most of the points (18 out of 26) lie in the first quadrant suggesting that most neurons preferred same direction of motion for both kind of stimuli. DSI for LM gratings is significantly greater than DSI for VM gratings (Wilcoxon signed rank one-tailed test, $\mathrm{p}<0.05$ ), suggesting that neurons had weaker direction selectivity for VM gratings compared to LM gratings.

These results show that neurons' responses are not to the local motion of the carrier in VM gratings but rather to the envelope in a form-cue invariant manner. If a neuron was responding to the carrier local motion then optimal envelope orientation for VM gratings would be approximately orthogonal to optimal orientation of LM gratings and histogram in Fig. 5B would peak around 90 deg instead of 0 deg.

\section{Carrier temporal frequency tuning}

A previous study (Mareschal \& Baker, 1998b), demonstrated that for most of the second-order responsive neurons in cat Area of 18 show bandpass tuning to the temporal frequency of drifting envelopes of CM gratings. Neurons were systematically selective for lower temporal frequencies for envelopes of $\mathrm{CM}$ gratings compared to LM gratings. Responses of these neurons usually fall off to spontaneous activity above an envelope temporal frequency of about $10 \mathrm{~Hz}$, while for luminance gratings, responses fall off around $16 \mathrm{~Hz}$. In order to see if neurons show similar tuning properties to drifting carriers, we measured responses for 
CM, and both "uni-directional" and "bi-directional" VM gratings, by varying carrier temporal frequency.

For "uni-directional" VM gratings one carrier was always held stationary and other drifted at varying temporal frequencies in both directions. Neurons showed diverse tuning properties for carrier temporal frequency. For some neurons, response decreased with increasing temporal frequency Fig.6 (A); response of some neurons increased with temporal frequency $(\mathrm{C})$; some neurons responded equally to all temporal frequencies (D); a few showed band-pass tuning (E), and some showed no such particular pattern (B, F). Interestingly, almost all neurons showed symmetric tuning i.e. similar response pattern for both directions of carrier motion indicated by values of symmetry indexes close to 1 in Fig. 6 . The scatterplot in Fig. 9A shows a given neuron's fall-off index for “unidirectional" VM gratings against fall-off index for LM gratings. Most of the points (19 out of 24) lie above the equality line suggesting that for "unidirectional" VM gratings neuronal responses fall off relatively less than LM responses at high temporal frequencies. The fall-off index values are significantly greater for VM gratings compared to LM gratings (Wilcoxon signed rank onetailed test, $\mathrm{p}<0.05)$.

For "bi-directional" VM gratings, the carrier gratings drifted with equal and opposite velocities. To measure carrier temporal frequency tuning for "bidirectional" VM gratings, temporal frequencies up to $16 \mathrm{~Hz}$ were tested. Fig. 7 shows carrier temporal frequency tuning for six neurons. The responses for these neurons decreased with increasing temporal frequency, except for one (Fig. 7F) 
which showed band-pass tuning. This was the only neuron that showed band-pass tuning in our sample of 19 neurons-usually they showed low pass responses.

In previous single-unit studies using $\mathrm{CM}$ stimuli, the carrier grating was always held stationary while the envelope was drifting over it (Zhou \& Baker, 1993, 1994; Mareschal \& Baker, 1998, 1999; Song \& Baker, 2007). In our present study, in order to measure dynamic properties of early stages of a filter-rectifyfilter model, we measured temporal frequency tuning for drifting carriers of $\mathrm{CM}$ gratings. Since we wanted to compare this CM carrier temporal frequency tuning with the carrier tuning for VM gratings, we fixed the carrier orientation perpendicular to the envelope orientation for $\mathrm{CM}$ gratings, and varied carrier temporal frequency from $0 \mathrm{~Hz}$ to $16 \mathrm{~Hz}(0 \mathrm{~Hz}$ corresponds to stationary carrier). Fig. 8 shows carrier temporal frequency tuning for six neurons. Neurons showed very diverse tuning, but most of them responded optimally to a grating with stationary carrier and response decreased with increase in temporal frequency. However some neurons responded quite well to very high temporal frequencies as well (Fig. 8 D, F). Similar to the results from "uni-directional” VM gratings, neurons showed symmetric tuning to carrier temporal frequency, i.e. the response pattern was similar to both directions of carrier motion, indicated by values of symmetry indexes close to 1 in Fig. 8. The scatter plot in Fig. 9 B shows a given neuron's fall-off index for CM gratings against fall-off index for LM gratings. There was no systematic relationship between fall-off indexes for these two gratings. 


\section{Responsiveness to uni-directional and bi-directional gratings}

In order to see if all $\mathrm{CM}$ grating responsive neurons also respond to both "uni-directional" and "bi-directional" VM gratings or only fraction of them respond to these two types of VM gratings, we compared response strength of $\mathrm{CM}$ responsive neurons for "uni-directional" and "bi-directional" VM gratings using their optimal parameters. The scatter plot in Fig. 10 A shows response strength to "bi-directional" vs "uni-directional" VM gratings for 19 neurons (5 simple and 14 complex) from carrier temporal frequency data like those in Figs.6 \& 7. All the data points on the plot either lie below the equality line or on it, indicating that neurons responded strongly to "uni-directional" compared to "bi-directional" VM gratings. Responses of the neurons to "uni-directional" are significantly greater than "bi-directional" VM gratings (Wilcoxon signed rank one-tailed test, $\mathrm{p}<$ 0.05). Even though all (19 out of 19) second-order responsive neurons responded significantly to "uni-directional" VM gratings, some (9 out of 19) failed to respond significantly (one tailed t-test) to "bi-directional" gratings. The histogram (Fig. 10 B) showing ratio of response strength to "bi-directional" and "unidirectional" demonstrates that for all neurons the ratio was either equal to or less than one and for 16 out of 19 neurons the ratio was less than 0.6 . Note that both types of VM gratings were not tested on all of the second-order responsive neurons due to inability to hold neurons for long recording durations. So this scatter plot contains only those second-order responsive neurons that were tested with both types of VM gratings. 


\section{Envelope spatial frequency tuning}

An interesting question is whether "uni-directional" and "bi-directional" VM gratings are detected due to dynamic discontinuities between the moving carriers, i.e. an "edge-based" processing, or by relative speeds of the carriers, i.e. a "region-based" processing. We measured envelope spatial frequency tuning curves to uni- and bi-directional VM gratings and compared them to the tuning for illusory contours (IC), which are thought to be detected by "edge-based" processing (Song \& Baker 2007). Interestingly, neurons showed distinct tuning for envelope spatial frequency of "uni-directional" and "bi-directional" VM gratings. Neurons showed similar envelope spatial frequency selectivity for ICs and "bi-directional" VM gratings (Figs. 11 A \& B). This suggests that bidirectional boundaries are detected in a manner like ICs i.e. by dynamic phasediscontinuities of the carrier textures along the boundary. However, neurons were selective for higher envelope spatial frequencies of "uni-directional" VM gratings compared to ICs, approximately two times the peak envelope spatial frequency for ICs (Figs. 11 A, B, C \& D). Therefore, uni-directional boundaries may be detected by both differences in the speeds of textures between envelope half-cycles, i.e. "region-based" processing and by dynamic discontinuities, i.e. "edge-based" processing. Also note that, peak spatial frequency for LM gratings was higher than or equal to peak spatial frequency for "uni-directional" VM gratings (Figs. 11 A, B, C \& D). 


\section{Chapter 4 - Discussion}

This study has demonstrated that neurons in cat visual cortex as early as Area 18 that respond to second-order stimuli such as contrast-defined contours can also respond to motion-defined contours. These neurons were selective to similar orientations of luminance- and motion-defined contours, as well as similar directions of motion "form-cue invariance", although the strength of direction selectivity was weaker for motion-defined contours compared to luminance contours. These neurons were also selective for spatial frequency of the carrier gratings used for making motion-defined contours. This carrier selectivity was very similar to the selectivity shown for the carrier of contrast-defined contours. These findings suggest that both kinds of contours are detected by the same nonlinear neural mechanism. For contrast- and motion-defined boundaries we also measured dependence of neurons' responses on temporal frequency of drifting carrier gratings, and found diverse tuning for the neurons. But for a given neuron, tuning was similar for both directions of carrier motion. Some neurons responded significantly to very high carrier temporal frequencies to which cortical neurons mostly fail to respond when tested with luminance gratings.

\section{Neural Mechanism}

A model consisting of two parallel streams (Fig. 12) has been proposed to explain responses of Area 18 neurons to first- and second-order stimuli (Zhou\&Baker, 1993,1996; Mareschal \& Baker,1998,1999; Song \& Baker, 2006, 2007). The first stream consists of a conventional coarse spatial scale linear filter (F0) selective for orientation, direction of motion and spatial frequency of 
luminance gratings. The second stream consists of a Filter-Rectify-Filter (FRF) model that can explain responses of neurons to second-order stimuli such as contrast- and texture-defined boundaries (Chubb \& Sperling, 1988; Wilson, 1999). This FRF model is composed of two linear filtering stages that are connected by nonlinearity (eg. rectification). The first stage consists of small scale spatial filters (F1) that are selective for high spatial frequencies of the carrier. The outputs of these early filters are rectified and pooled by a coarse spatial scale late filter (F2), which is selective for envelope orientation, direction of motion and spatial frequency. Filters F0 and F2 have similar preference for orientation and direction of motion, but spatial frequency selectivity is coarser for F2 compared to F0. Here we explore whether the FRF model proposed to explain responses to contrast- and texture-defined boundaries can also explain responses of Area 18 neurons to motion-defined boundaries.

If motion-defined boundaries are processed by a common FRF like mechanism, then neurons should show similar tuning properties for carrier and envelope of motion- and contrast-defined boundaries. In processing of motiondefined boundaries by an FRF model, filters F1 could act as local motion detectors whose outputs will be rectified and pooled by filter F2. If these F1 filters are common for processing motion- and contrast-defined boundaries, then neurons should have similar carrier spatial frequency selectivity for both kinds of boundaries. Our results demonstrate that a given neuron is indeed selective for similar carrier spatial frequency for motion- and contrast-defined gratings (Fig. 2). 
A FRF model of the above kind could detect VM grating stimuli in two ways. The neuron could be responding to differences in the speeds of drifting carriers between adjacent half cycles of VM gratings (a "region-based" processing) and/or to the dynamic discontinuities (carriers in adjacent half-cycles moving in and out of phase with one another) along the boundaries (an "edgebased" processing) similar to illusory contours (Song \& Baker 2007). Since dynamic phase discontinuities are present in both "uni-directional" and "bidirectional" VM gratings, an FRF model could produce an edge-based response to both stimuli. For this model to respond in a "region-based" manner to "unidirectional" VM gratings, its early F1 filters must respond differently to stationary and moving carriers. For "bi-directional" VM gratings, an FRF model will give a region-based response only if its early filters can distinguish between carriers drifting with equal speeds in opposite directions, and this is possible only if the early filters are selective for motion direction.

We measured temporal tuning properties of early filters by systematically varying the temporal frequency of a drifting carrier grating for contrast-defined boundary stimuli. The neurons' responses were symmetric for both directions of carrier motion, suggesting that early filters of the FRF model are not direction selective. For most neurons, response peaked when the carrier was held stationary, and gradually declined with increasing carrier temporal frequency. Since early filters of the FRF model are not direction selective, the FRF model would predict symmetric carrier temporal frequency tuning for both directions of carrier motion for "uni-directional” VM gratings, which our results (Fig.6) demonstrate. The 
model would also predict that responses to "uni-directional" VM gratings would be mediated by both "edge-based" processing (because of dynamic discontinuities) and "region-based" processing (because early filters give different response to stationary and moving carrier). Responses to "bi-directional" VM gratings would be mediated by "edge-based" processing only, and not by "regionbased" processing (because early filters are not direction selective).

To test these predictions about mechanism, we measured envelope spatial frequency tuning for both "uni-directional" and "bi-directional" VM gratings, and compared it with envelope spatial frequency tuning for illusory contours (ICs). ICs are thought to be detected in an "edge-based" manner (Wilson, 1999; Song \& Baker, 2007), so if a VM grating is also detected in an "edge-based" manner it will show the same tuning for envelope spatial frequency as an IC. But if a VM grating is detected by "region-based" processing (like contrast-defined boundaries (Song \& Baker, 2007)) it will be tuned to envelope spatial frequency twice that of IC, as there are two phase-discontinuity edges in one envelope cycle of IC. Our results show that "bi-directional" VM grating responses are tuned to the same envelope spatial frequencies as illusory contours. But "uni-directional" VM grating responses are tuned to spatial frequencies higher than the optimal for illusory contours, but less than twice this optimal value. This result suggests that "uni-directional" gratings are detected by a mixture of both "region-based" and "edge-based" processing. Also our results (Fig. 10) show that responses of neurons are stronger to "uni-directional" gratings compared to "bi-directional" gratings, and this could be because "uni-directional" gratings are simultaneously 
detected by both "region-based" and "edge-based" processing, while "bidirectional" gratings are detected by "edge-based" processing only. Predictions made from the FRF model for neural responses to VM gratings were in accordance to the actual neuronal responses, suggesting that VM gratings are indeed detected by the same FRF model that detects CM gratings.

\section{Sinusoidal grating carrier}

It might seem counterintuitive to use sinusoidal gratings as a carrier instead of random dot texture patterns used in previous studies (Chaudhuri \& Albright, 1997; Mysore et al. 2006; Sary et al. 1993, 1995; Zeki et al., 2003), as these patterns look more similar to texture patterns present in the real world. A sinusoidal grating carrier with spatial frequency outside a neuron's luminance passband provides powerful advantages in ruling out simple linear/luminance artifacts. Firstly, it ensures that the responses are not mediated by the same linear filter thought to process luminance gratings. A random dot texture patterns however, are broadband in spatial frequency and some of its energy might fall within the luminance passband of a neuron, giving rise to a linear response. Secondly, these neurons show narrow carrier spatial frequency tuning, and this result rules out the possibility that their responses are mediated by early nonlinearities of the retina or CRT because such nonlinearities would not predict selectivity for carrier spatial frequencies.

However VM gratings with sinusoidal grating carriers introduce an ambiguity as to what the neuron is actually responding to. If a snapshot image of VM stimuli is taken at some moment in time (Fig. 1 C) then it looks like an 
illusory contour with phase-discontinuities between two carriers. But measuring envelope spatial frequency tuning to VM gratings as described above, we were able to disambiguate between responses to phase discontinuities and difference in speeds of carriers. Note that in natural scenes, shear motion between textures will give rise to both of these cues: phase discontinuities and relative speed.

\section{Cue-invariance}

For the visual system to perform figure-ground segregation, it should be able to recognize an object from a background which might be the same with respect to all cues except one, e.g. color, but when the object is surrounded by some other background it might have the same color as the background and differ in its texture. In order for the visual system to perform this task, information about the presence of a particular cue is not important, but rather the contrast between cues that distinguish an object from its background is of primary importance. So the visual system should be able combine information across different cues to perform figure-ground segregation, i.e. the segmentation mechanism needs to be form-cue invariant. This strategy of form cue-invariance is computationally economical, and can help resolve perceptual ambiguities when multiple cues are present. In addition it might be important for shape recognition and shape constancy.

Neurons in the early visual cortex have been previously shown to respond in a form-cue invariant manner to stimulus attributes such as orientation and motion direction of boundaries. Neurons in cat Area 17 (Zhou \& Baker, 1993, 1996), cat Area 18 (Zhou \& Baker, 1993, 1996; Mareschal \& Baker, 1998a, 
1998b; Leventhal et al., 1998; Zhou et al. 2001; Sheth et al., 1996; Song \& Baker, 2006,2007; Tanaka \& Ohzawa, 2006), primate V1 (Chaudhuri \& Albright, 1997) and primate V2 (von der Heydt et al., 1984; von der Heydt \& Peterhans, 1989; Leventhal et al., 1998; Lui et al., 2005) have been shown to respond in a form-cue invariant manner to the orientation of luminance and non-luminance boundaries. In this study, we show that neurons in cat Area 18 that have been previously shown to selectively respond to luminance-, texture- and contrast-defined boundaries can also respond to motion-defined boundaries in a form-cue invariant manner. These neurons showed similar orientation selectivity for luminance- and motion-defined boundaries, but direction selectivity was weaker to motiondefined boundaries, similar what was found previously for contrast- and texturedefined boundaries (Song \& Baker, 2006, 2007). This form-cue invariant orientation tuning in early visual cortex could be utilized by higher brain areas like V4 and IT that respond to more complex stimuli in a form-cue invariant manner (Mysore et al., 2006; Sary et al., 1993).

(Born \& Tootell, 1992; Born, 2000) found neurons in primate area MT and (Shen et al., 2007) found neurons in primate area V1 that responded to relative motion stimuli. But these responses were fundamentally different from what we report here, because those neurons responded to differences in motion of the stimulus between classical and non-classical receptive fields. Also, these neurons would not show form-cue invariant orientation selectivity to motion-defined boundaries. Regardless, these neurons could be useful in figure-ground 
segregation, and might act as subunits for neurons that detect orientation of motion-defined boundaries.

A neuroimaging study in human subjects (Reppas et al., 1997) found that strong motion boundary selective signals are present in early areas like V1 and V2. One might think that our results are in agreement with theirs, but it is difficult to make a direct comparison between single-unit and neuroimaging studies. If a certain brain area in a neuroimaging study is responding significantly greater to motion boundary stimuli compared to uniform or transparent motion, it doesn't necessarily imply that neurons in that particular brain area are selective for orientation of motion boundaries. Neurons could be responding by centresurround antagonistic mechanism (Born \& Tootell, 1992; Born, 2000; Shen et al., 2007). However, a recent study (Larsson et al., 2010) was able to demonstrate orientation selectivity to motion boundaries in human visual cortex using an event-related fMRI adaptation technique. They showed that most of the motion boundary responsive visual areas like V2, V3, V3A, V3B, LO1, LO2, hV4 and V7 identified in previous neuroimaging studies (Dupont et al. 1997; Larsson and Heeger 2006; Tyler et al. 2006; Van Oostende et al. 1997; Zeki et al., 2003) are orientation selective. These results argue against the initial notion from neuroimaging studies (Dupont et al. 1997; Van Oostende et al. 1997) that motion boundaries are processed in a specialized brain area, "kinetic occipital" or KO (corresponding to LO1, LO2, and V3B). 


\section{Chapter 5 - Summary \& Conclusion}

This study has demonstrated that neurons in cat visual cortex as early as Area 18 , that have been previously shown to respond to second-order stimuli such as contrast-defined boundaries, can also respond to motion-defined boundaries. These neurons are selective to similar orientations of luminance- and motiondefined contours, as well as similar directions of motion- i.e. "form-cue invariance". These neurons show similar spatial frequency selectivity for carrier gratings used for constructing contrast- and motion-defined boundaries. These neurons show diverse tuning for carrier temporal frequency of contrast- and motion-defined boundaries - but for a given neuron, temporal frequency tuning is similar for both directions of carrier motion. Neurons respond significantly to motion-defined boundaries with very high carrier temporal frequencies, but for luminance boundaries at these high temporal frequencies responses of most neurons fall to spontaneous activity.

This study suggests that specific processing of motion-defined boundaries begins in early visual areas. Further, it suggests that these boundaries are processed by a common nonlinear mechanism that processes other second-order stimuli such as contrast-and texture-defined boundaries. The neuronal responses to motion-defined boundaries can be explained by the same FRF model previously used to explain responses to contrast-defined boundaries. The carrier temporal frequency tuning results suggests that the early filters of this model, that are designed to detect carrier properties, are not selective to motion direction. 


\section{References}

Albright, T. D., (1992) Form-cue invariant motion processing in primate visual cortex. Science, 255;1141-1143.

Baker, C.L., and Mareschal I. (2001). Processing of second-order stimuli in the visual cortex. Prog Brain Res, 134: 171-191.

Born, R.T., Tootell, R.B.H., (1992). Segregation of global and local motion processing in primate middle temporal visual area. Nature, 357; 497-499.

Born, R.T. (2000). Center-surround interactions in the middle temporal visual area of the owl monkey. Journal of Neurophysiology, 84: 2658-2669.

Brainard, D. H., (1997). The psychophysics toolbox. Spatial Vision, 10: 433-436.

Chaudhuri, A. and Albright, T. D. (1997). Neuronal responses to edges defined by luminance vs. temporal texture in macaque V1. Visual Neuroscience 14:949962.

Chubb, C. and Sperling, G. (1988). Drift balanced random stimuli: a general basis for studying non-Fourier motion perception. J. Opt. Soc. Am., 5:1986-2007.

DeAngelis, C. G., Freeman, R. D., and Ohzawa, I. (1994). Length and width tuning of neurons in the cat's primary visual cortex. Journal of Neurophysiology, 71: 347374.

Efron, B. \& Tibshirani, B. (1993). An introduction to bootstrap. New York: Chapman \& Hall.

Hubel DH and Wiesel TN. (1962). Receptive fields, binocular interaction and functional architecture in cat's visual cortex. Journal of Physiology 160:106-154.

Larsson, J., Heeger, D.J., \& Landy, M.S. (2010). Orientation selectivity of motionboundary responses in human visual cortex. Journal of Neurophysiology 104: 2940-2950.

Ledgeway, T. \& Smith, A. T. (1994). Evidence for separate motion-detecting mechanisms for first- and second-order motion in human vision. Vision Research, 34:27272740 .

Leventhal, A.G., Wang, Y., Schmolesky, M.T. \& Zhou, Y. (1998). Neural correlates of boundary perception. Visual Neuroscience, 15:1107-1118.

Lui, L.L., Bourne, J.A. \& Rosa, M.G. (2005). Single unit responses to kinetic stimuli in New World monkey area V2: Physiological characteristics of cue-invariant neurons. Experimental Brain Research, 162:100-108. 
Marr, D. (1982) Vision. Freeman, San Francisco.

Marcar, V.L., Raiguel, S.E., Xiao, D., and Orban, G.A. (2000) Processing of kinetically defined boundaries in Areas V1 and V2 of the Macaque Monkey. Journal of Neurophysiology 84: 2786-2798.

Mareschal I., Baker, C.L. (1998a). A cortical locus for the processing of contrast-defined contours. Nature Neuroscience 1:150-154.

Mareschal I., Baker CL Jr. (1998b) Temporal and spatial response to second-order stimuli in cat area 18. Journal of Neurophysiology, 80: 2811-2823.

Mareschal I, Baker CL Jr. (1999). Cortical processing of second-order motion. Visual Neuroscience, 16: 527-540.

Marida, K.V. (1972). Statistics of directional data. New York: Academic Press.

Mather, G. , \& West, S. (1993). Evidence for second-order motion detectors. Vision Research, 33:1109-1112.

Mysore, S.G., Vogels, R., Raiguel, S. E. and Guy, A. (2006) Orban Processing of Kinetic Boundaries in Macaque V4. Journal of Neurophysiology, 95:1864-1880.

Nishida, S., Ledgeway, T., \& Edwards, M. (1997) Dual multiple-scale processing for motion in the human visual system. Vision Research, 37:2685-2698.

Pelli, D. G. (1997). The VideoToolbox software for visual psychophysics: Transforming numbers into movies. Spatial Vision, 10:437-442.

Regan D. (1989). Orientation discrimination for objects defined by relative motion and objects defined by luminance contrast. Vision Res. 29: 1389-1400.

Regan D and Hamstra SJ. (1992) Dissociation of orientation discrimination from form detection for motion-defined bars and luminance-defined bars: effects of dot lifetime and presentation duration. Vision Res. 32: 1655-66.

Reppas, J.B., Niyogi, S., Dale, A.M., Sereno,M.I., Tootell, R.B.H.(1997) Representation of motion boundaries in retinotopic human visual cortical areas. Nature, 388, 175-179.

Rogers, B. and Graham, M. (1979) Motion parallax as an independent cue for depth perception. Perception, 8: 125-134.

Sary, G., Vogels, R. and Orban G.A. (1993) Cue-invariant shape selectivity of macaque inferior temporal neurons. Science, 260: 995-997. 
Sary, G., Vogels, R. and Orban G.A. (1994) Orientation discrimination of motion defined gratings. Vision Research, 34:1331-1334.

Scott-Samuel, N.E., \& Georgson, M.A. (1999). Does early non-linearity account for second-order motion? Vision Research, 39: 2853-2865.

Sheth, B.R., Sharma, J., Rao, S.C. \& Sur, M. (1996). Orientation maps of subjective contours in visual cortex. Science, 274, 2110-2115.

Shen, Z., Xu, W. and Li, C. (2007). Cue-invariant detection of centre-surround discontinuity by V1 neurons in awake macaque monkey. Journal of Physiology, 538.2, 581-592.

Skottun, B.C. (1994). Illusory contours and linear filters. Experimental Brain Research, 100:360-364.

Song, Y. and Baker CL Jr. (2007) Neuronal response to texture- and contrast-defined boundaries in early visual cortex. Visual Neuroscience, 24: 65-77.

Tanaka H., Ozhawal. (2006). Neural basis for stereopsis from second-order contrast cues. Journal of Neuroscience, 26:4370-4382.

von der Heydt, R., Peterhans, E. \& Baumgartner, G. (1984). Illusory contours and cortical neurons responses. Science, 244, 1260-1262.

von der Heydt, R., and Peterhans, E. (1989). Mechanisms of contour perception in monkey visual cortex. I. Lines of pattern discontinuity. Journal of Neuroscience, 9, 1731-1748.

Wilson, H. (1999) Non-Fourier cortical processes in texture, form, and motion perception. Cerebral Cortex.

Zeki S., R.J. Perry and A. Bartels. (2003) The Processing of Kinetic Contours in the Brain. Cerebral Cortex, 13:189-202.

Zhan C. A. and Baker CL Jr. (2006) Boundary cue invariance in cortical orientation maps. Cerebral Cortex 16: 896-906.

Zhou Y.X. \& Baker C.L. (1993). A processing stream in mammalian visual cortex neurons for non-Fourier responses. Science, 261:98-101.

Zhou Y.X. \& Baker C.L. (1996). Spatial properties of envelope-responsive cells in area 17 and18 neurons of the cat. Journal of Neurophysiology, 75:1038-1050. 
Figure 1. Four types of grating stimuli used in this study. (A) Luminance modulation (LM) sinusoidal grating with vertical orientation. (B) Contrast modulation (CM) grating with vertically oriented sinusoidal envelope that modulates the contrast of a horizontal high spatial frequency carrier grating. (C) "Uni-directional" velocity modulation (VM) grating with vertically oriented squarewave envelope that modulates the velocity of a horizontal high spatial frequency carrier grating. For "uni-directional" VM, the carrier in half of the envelope cycles is stationary, and in the other half it drifts with a specified temporal frequency. (D) "Bi-directional" velocity modulation (VM) grating is constructed similarly to "uni-directional" VM except that the carrier in alternate half cycles of the envelope drifts with equal speeds but in opposite directions. 
A
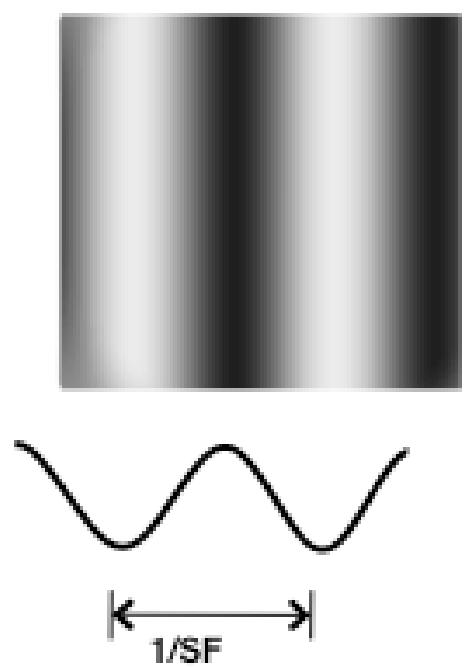

Luminance Modulation (LM)

C
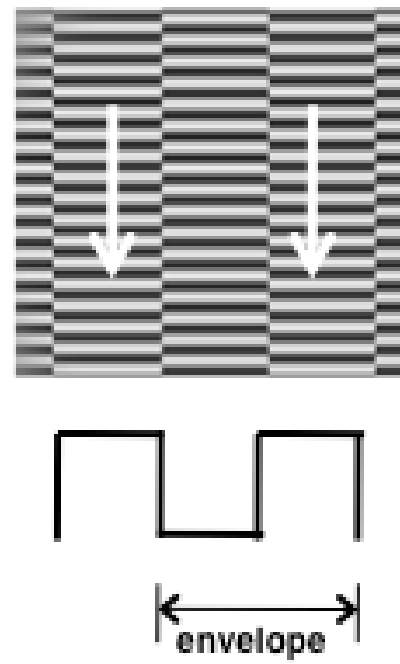

"Uni-Directional" Velocity Modulation (VM)
B
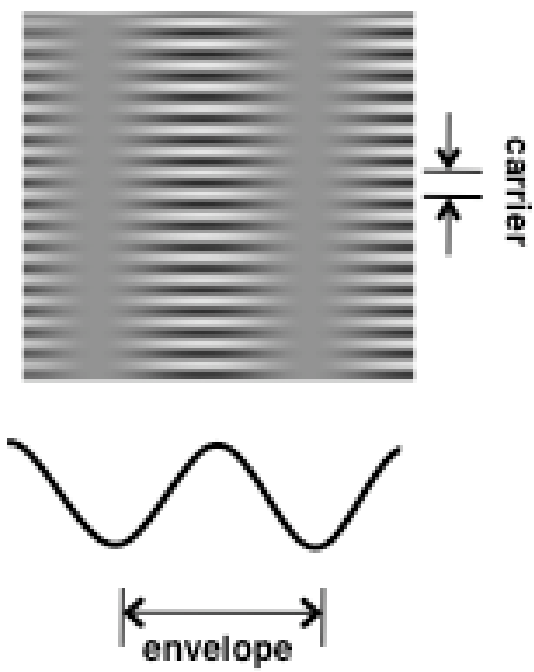

Contrast Modulation (CM)

D
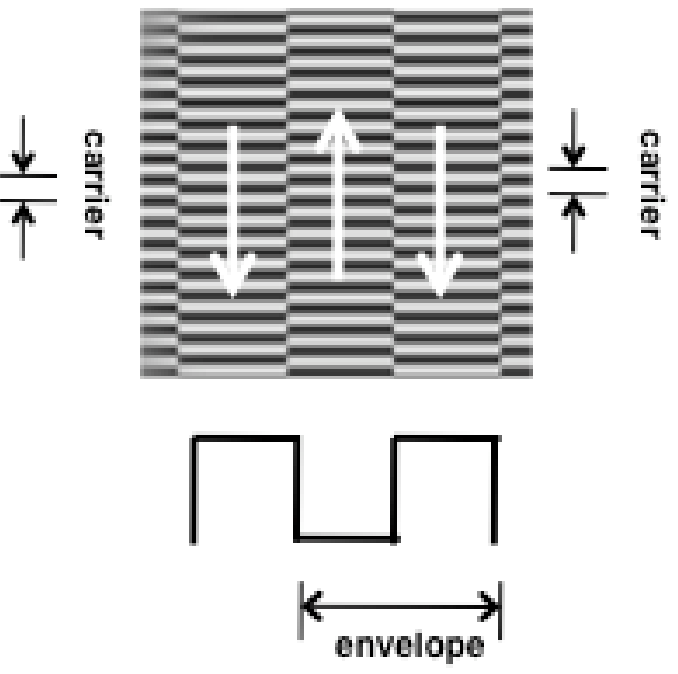

"Bi-Directional"

Velocity Modulation (VM) 
Figure 2. Responses of a typical neuron to luminance modulation (LM), contrast modulation (CM) and velocity modulation (VM) gratings. Neuronal responses to LM gratings are shown as a function of spatial frequency (grey diamonds) measured at grating's optimal orientation. Snapshots of luminance gratings with two different spatial frequencies are shown at the top. Optimal luminance spatial frequency for this neuron was $0.08 \mathrm{cpd}$, and neuronal response fell to spontaneous activity (dashed line) at spatial frequency of $0.3 \mathrm{cpd}$. Responses of the same neuron are measured to $\mathrm{CM}$ and VM gratings as a function of carrier spatial frequency, with envelope orientation kept the same as grating's optimal orientation and envelope spatial frequency slightly lower than optimal luminance spatial frequency. Snapshots of CM and VM gratings with two different carrier spatial frequencies are shown at the bottom. Carrier spatial frequency tuning for both gratings was very similar, with peaks around $0.8 \mathrm{cpd}$, much greater than the optimal luminance grating spatial frequency of $0.08 \mathrm{cpd}$. 

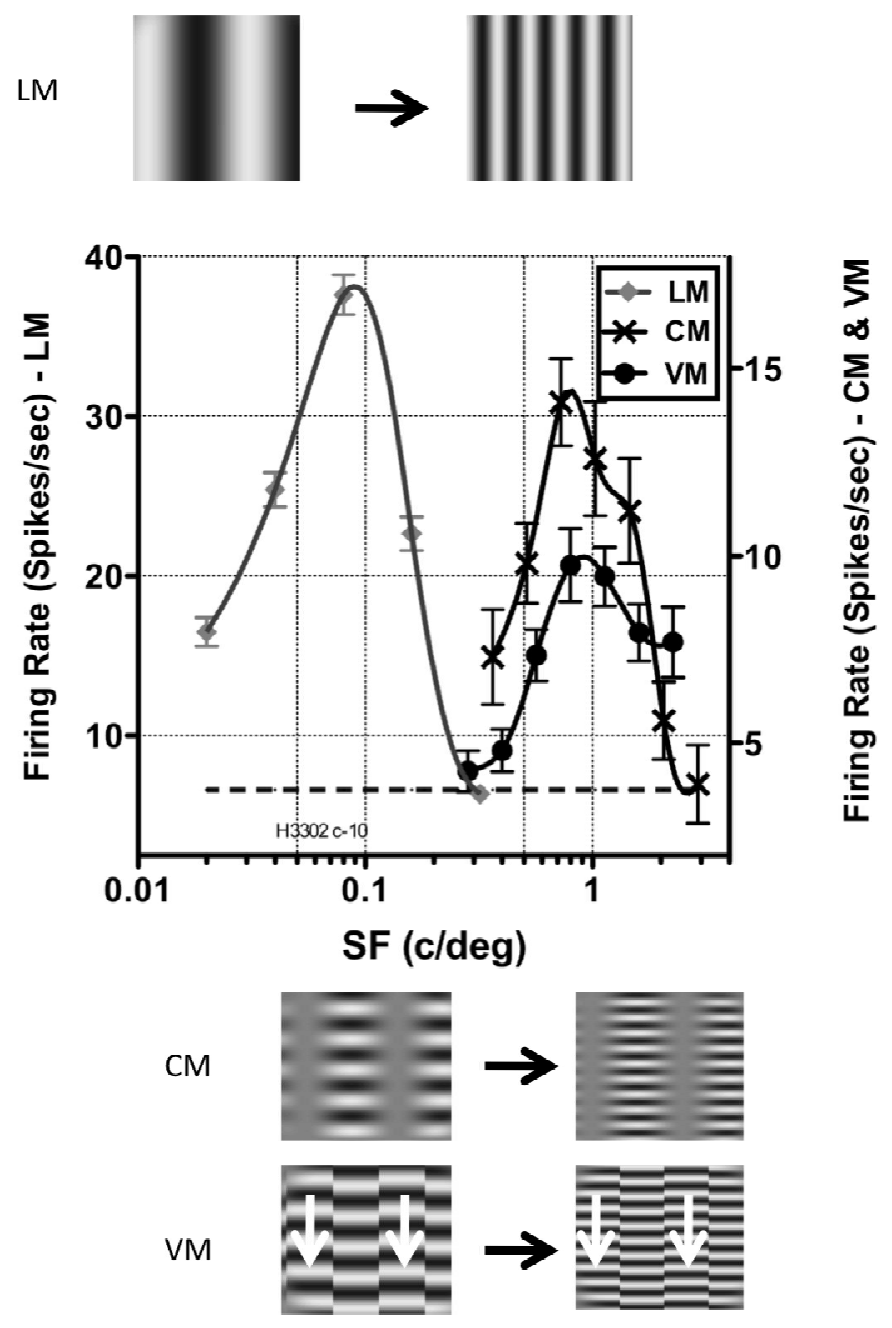

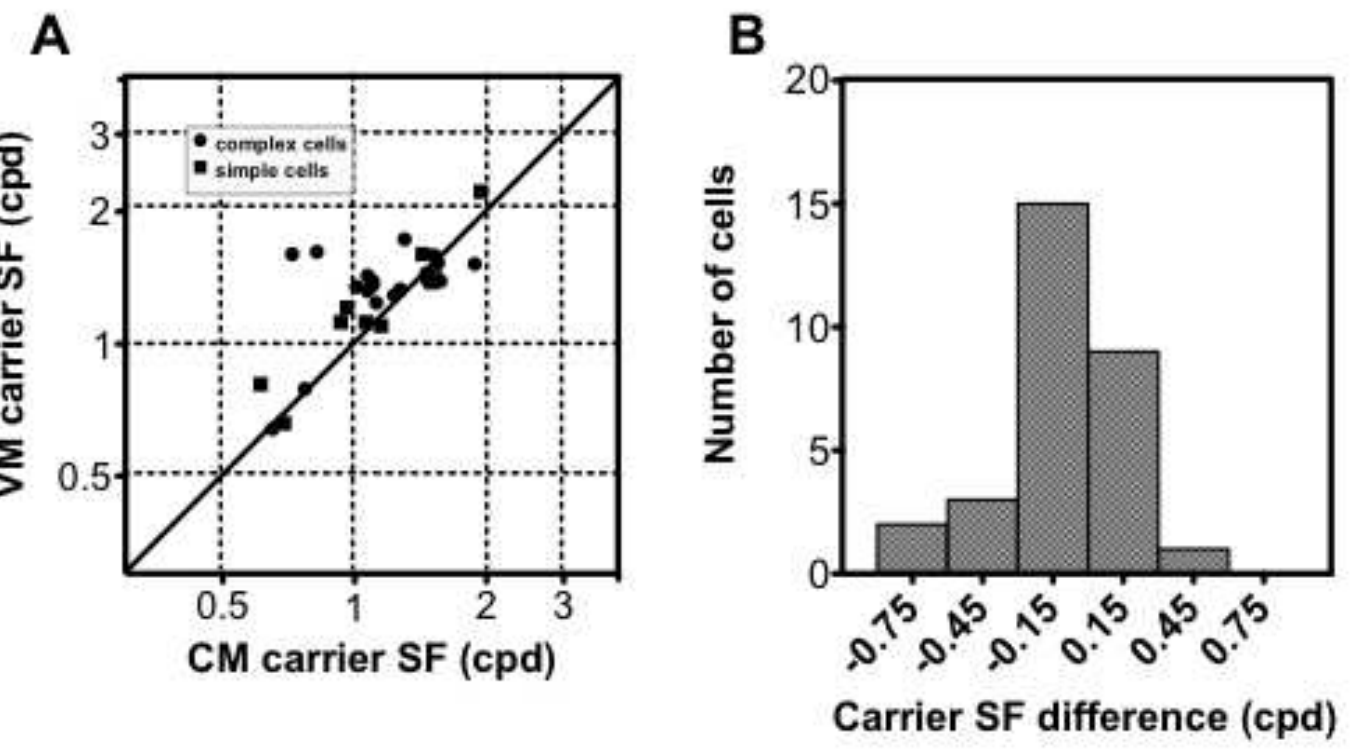

Figure 3. Relationship between optimal carrier spatial frequency for VM and CM stimuli. A, Scatter plot showing neurons' optimal carrier spatial frequency for VM gratings vs. CM gratings for 30 neurons ( 21 complex and 9 simple cells). A given neuron's optimal carrier spatial frequency for VM gratings is highly correlated with that for CM gratings $(r=0.7, p<0.0001) B$, histogram showing differences between optimal carrier spatial frequencies for CM and VM gratings. 
Figure 4. Orientation tuning of a typical neuron to LM gratings and envelope of VM and CM gratings. In these polar plots distance from the origin indicates neural response (spikes/sec); angular subtense represents envelope orientation (0-360 degrees). Snapshots of LM, VM and CM gratings at three different orientations are shown along with polar plots. Optimal orientation, circular variance (CV) and direction selectivity index (DSI) are shown at the bottom of each polar plot. This neuron showed similar orientation tuning and direction selectivity for three stimuli. 

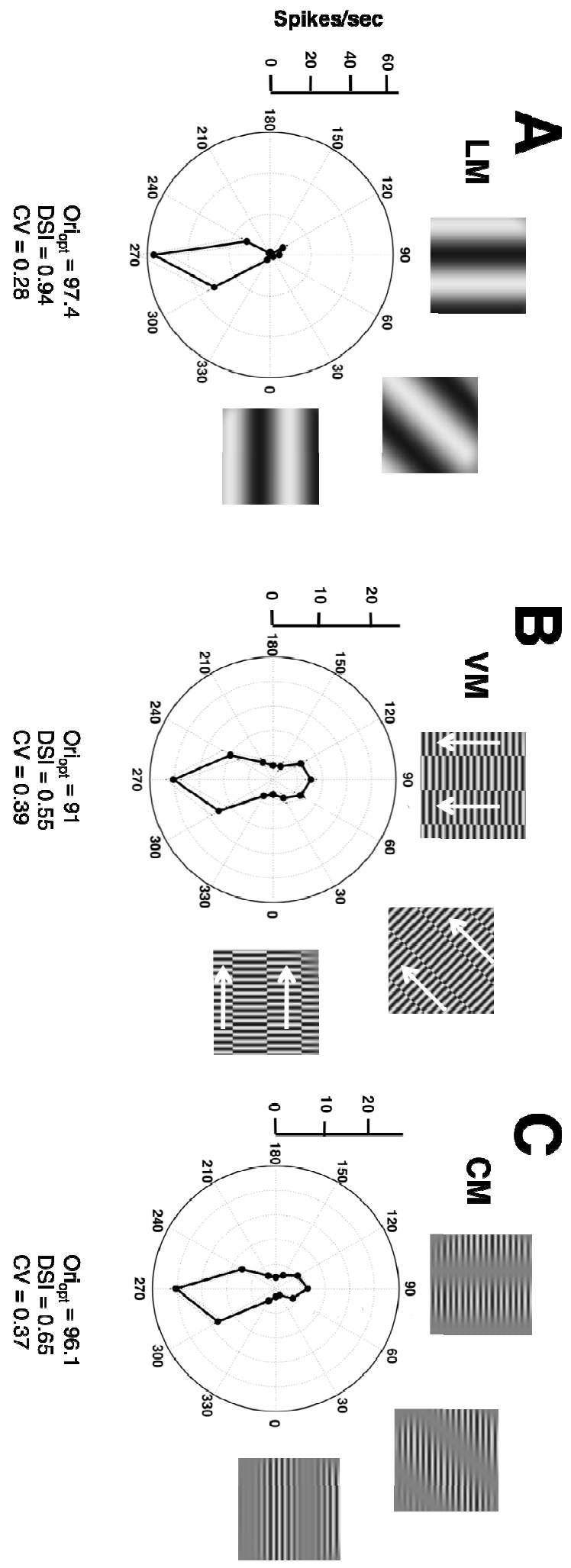
Figure 5. Relationship between orientation and direction selectivity for VM and LM stimuli, for a sample population of neurons. A, Scatter plot showing neurons' optimal envelope orientation for VM gratings vs. optimal orientation for LM gratings for 26 neurons (10 simple and 16 complex cells). A given neuron's optimal envelope orientation for VM gratings is highly correlated with optimal orientation for LM gratings $(r=0.95, p<0.0001)$ B, Histogram showing differences between optimal orientations for VM and LM gratings. C, Scatter plot showing a given neuron's envelope orientation circular variance (CV) for VM gratings vs. orientation circular variance (CV) for LM gratings. Most of the points lie above the equality line, indicating broader tuning for envelope orientation of VM gratings compared to orientation tuning for LM gratings. D, Scatter plot showing a given neuron's direction selectivity index for motion direction of envelope of VM gratings vs. motion direction of LM gratings. Most of the neurons preferred the same direction of motion for LM and VM gratings (18/26), while remaining neurons that preferred opposite directions were weakly direction selective to at least one of the two gratings. 
A

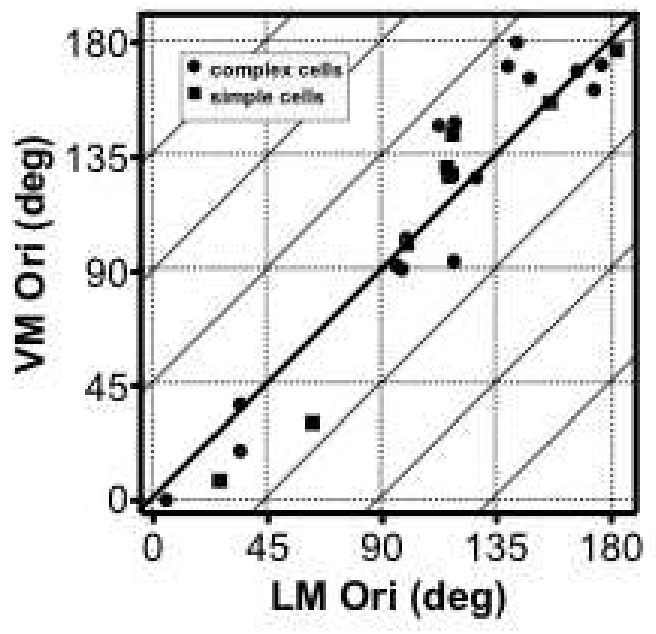

C

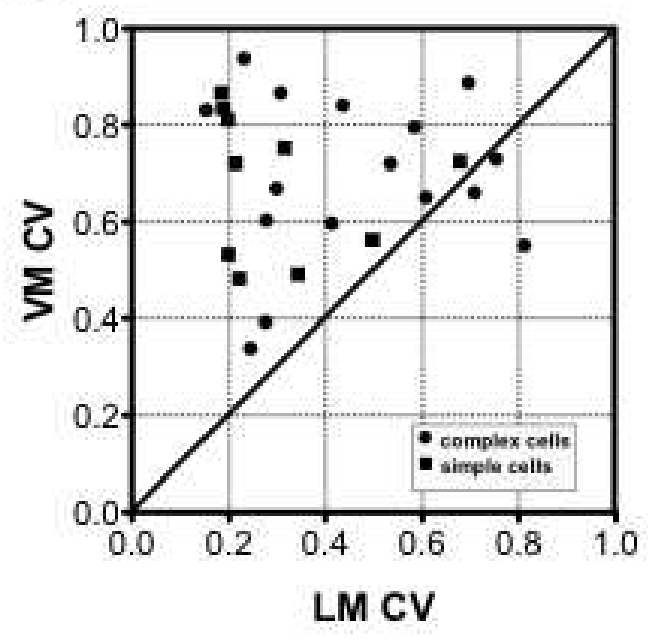

B

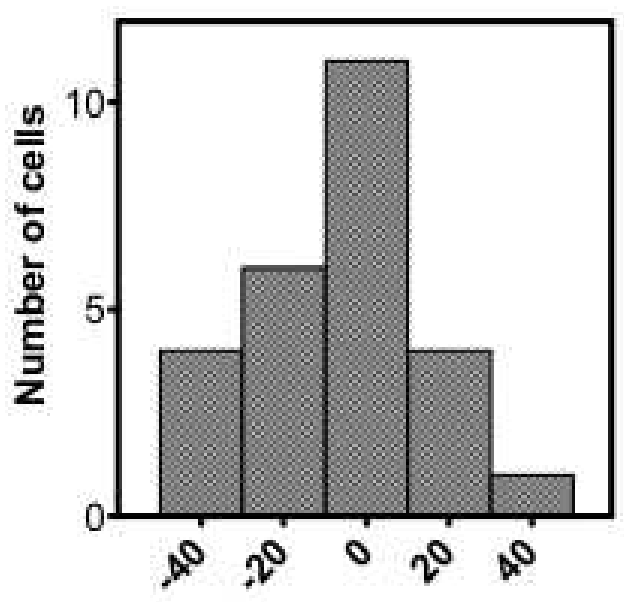

Ori difference (deg)

D

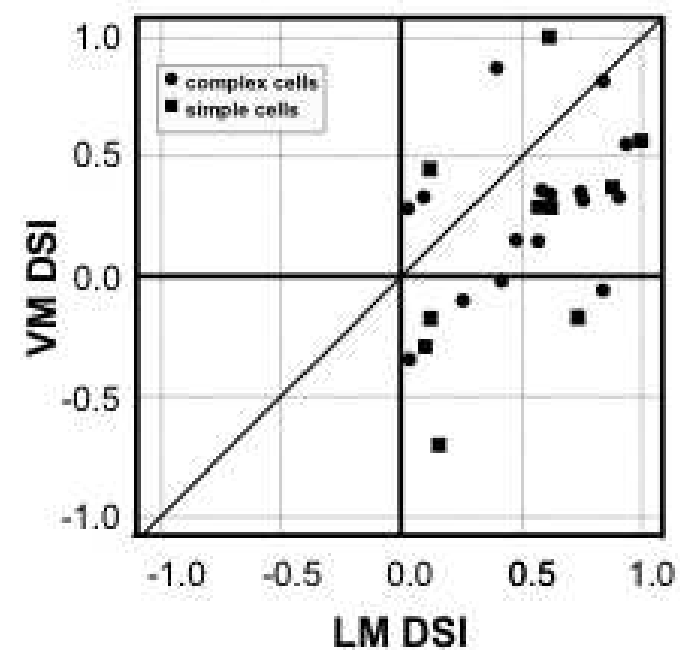


Figure 6. Carrier temporal frequency responses to "uni-directional" VM gratings, for six neurons. Neurons showed diverse tuning properties to carrier temporal frequencies, but most responded significantly to almost all temporal frequencies tested and tuning was similar for both directions of carrier motion. Negative value of carrier temporal frequency means carrier motion in opposite direction to that of positive values. Dashed lines indicate spontaneous activity. Symmetry index (SI) values represent symmetry of responses to both directions of carrier motion and fall-off index (FI) values represent relative fall in response at carrier temporal frequency of $16 \mathrm{~Hz}$ compared to maximum response are shown on the top of each plot. 

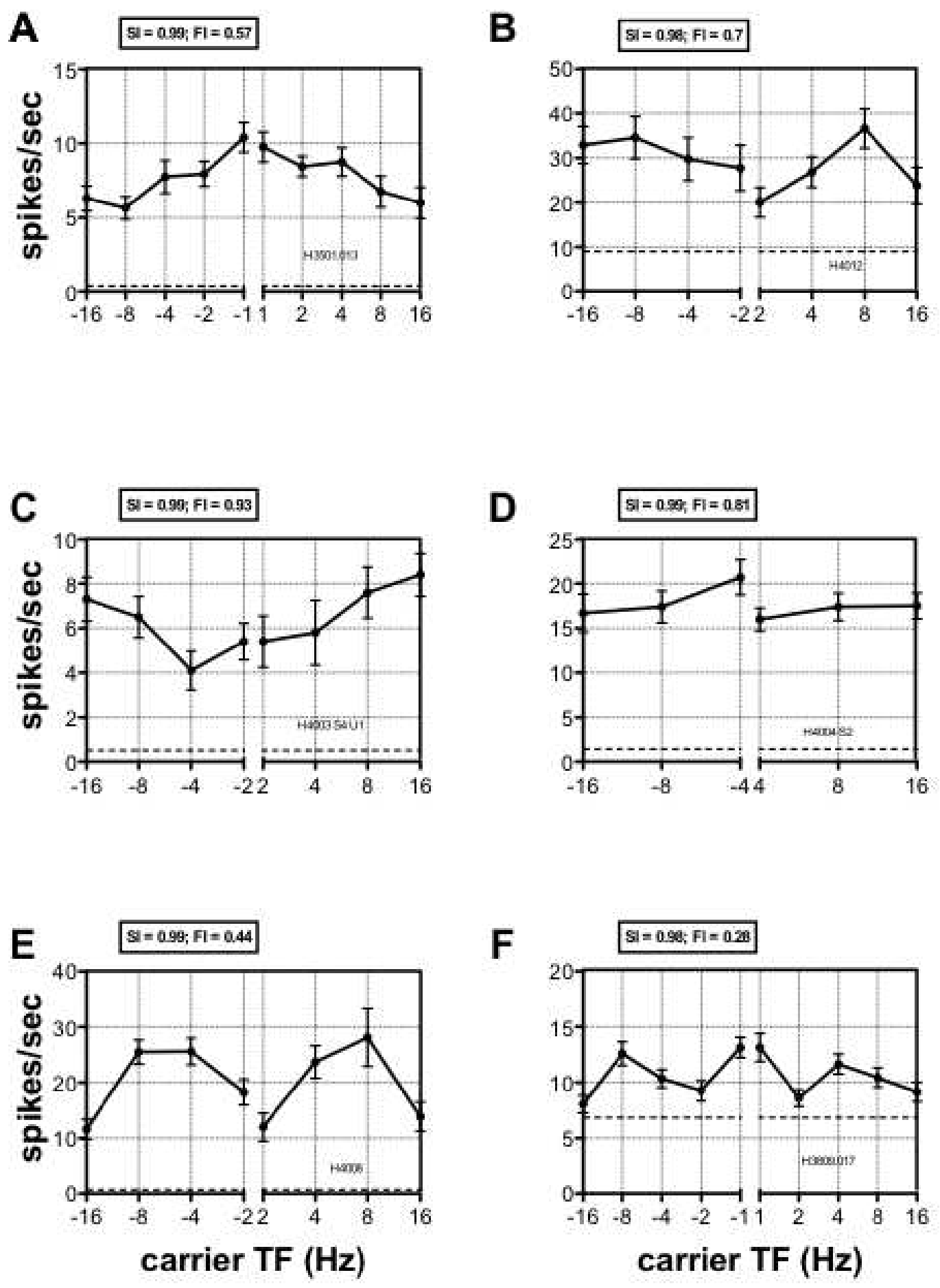
Figure 7. Carrier temporal frequency responses to "bi-directional" VM gratings, for six neurons. Responses typically $(A-E)$ decreased with increasing carrier temporal frequency, while neuron (F) showed band-pass tuning. Dashed lines indicate spontaneous activity. Fall-off index (FI) values are shown at the top of each plot. 

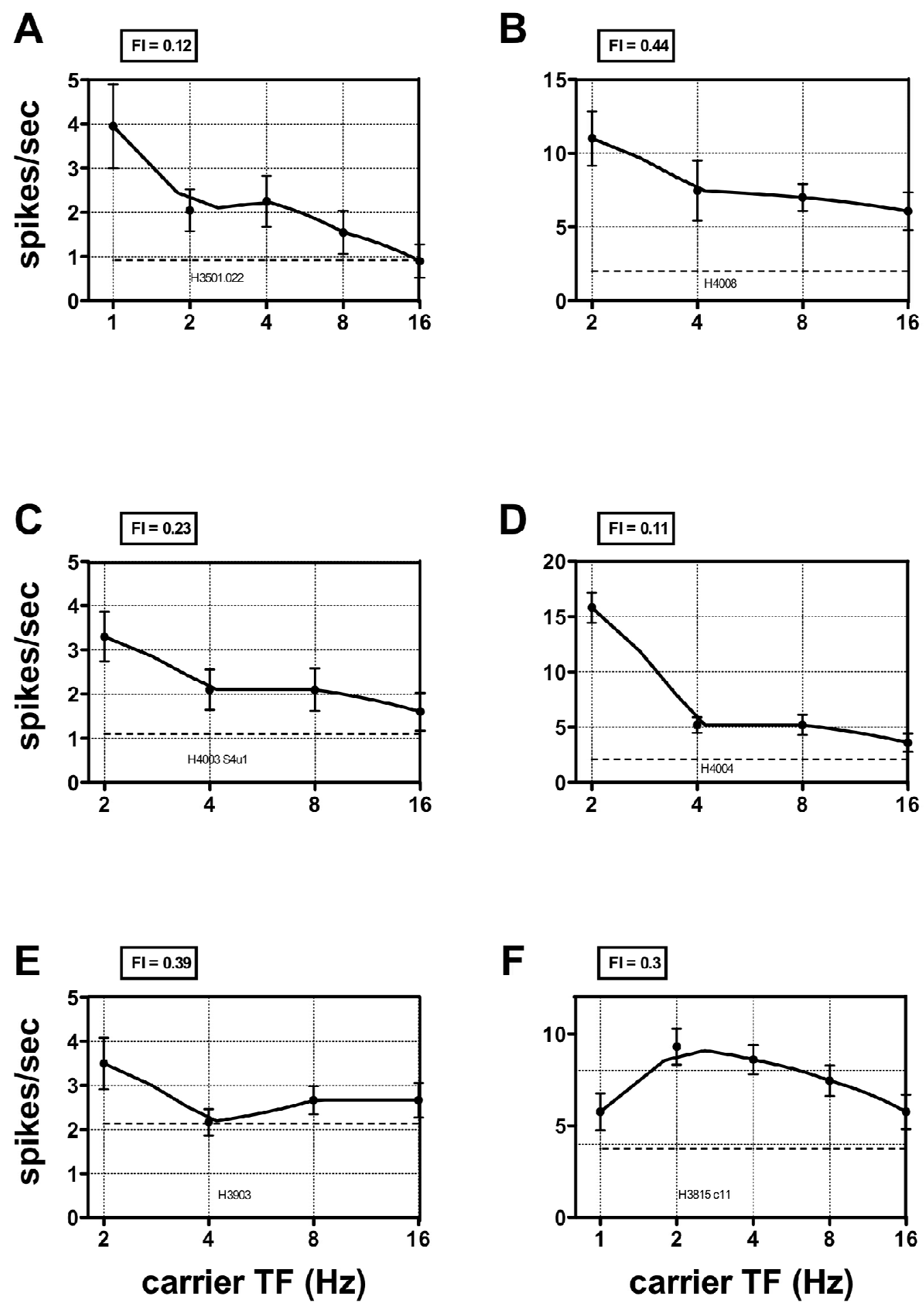
Figure 8. Carrier temporal frequency responses to CM gratings, for 6 neurons. Orientation of the carrier grating was kept perpendicular to the envelope orientation, and temporal frequency was varied from $\mathrm{OHz}$ (stationary carrier) to $16 \mathrm{~Hz}$ in both directions. Neurons responded optimally to a stationary carrier, and response typically decreased with increasing carrier temporal frequency, though some neurons responded equally well to drifting carriers (E, F). Neurons showed similar tuning for both directions of carrier motion. Negative value of carrier temporal frequency means motion in opposite direction to that of positive values. Dashed lines indicate spontaneous activity. Symmetry index (SI) values and fall-off index (FI) values are shown at the top of each plot. 

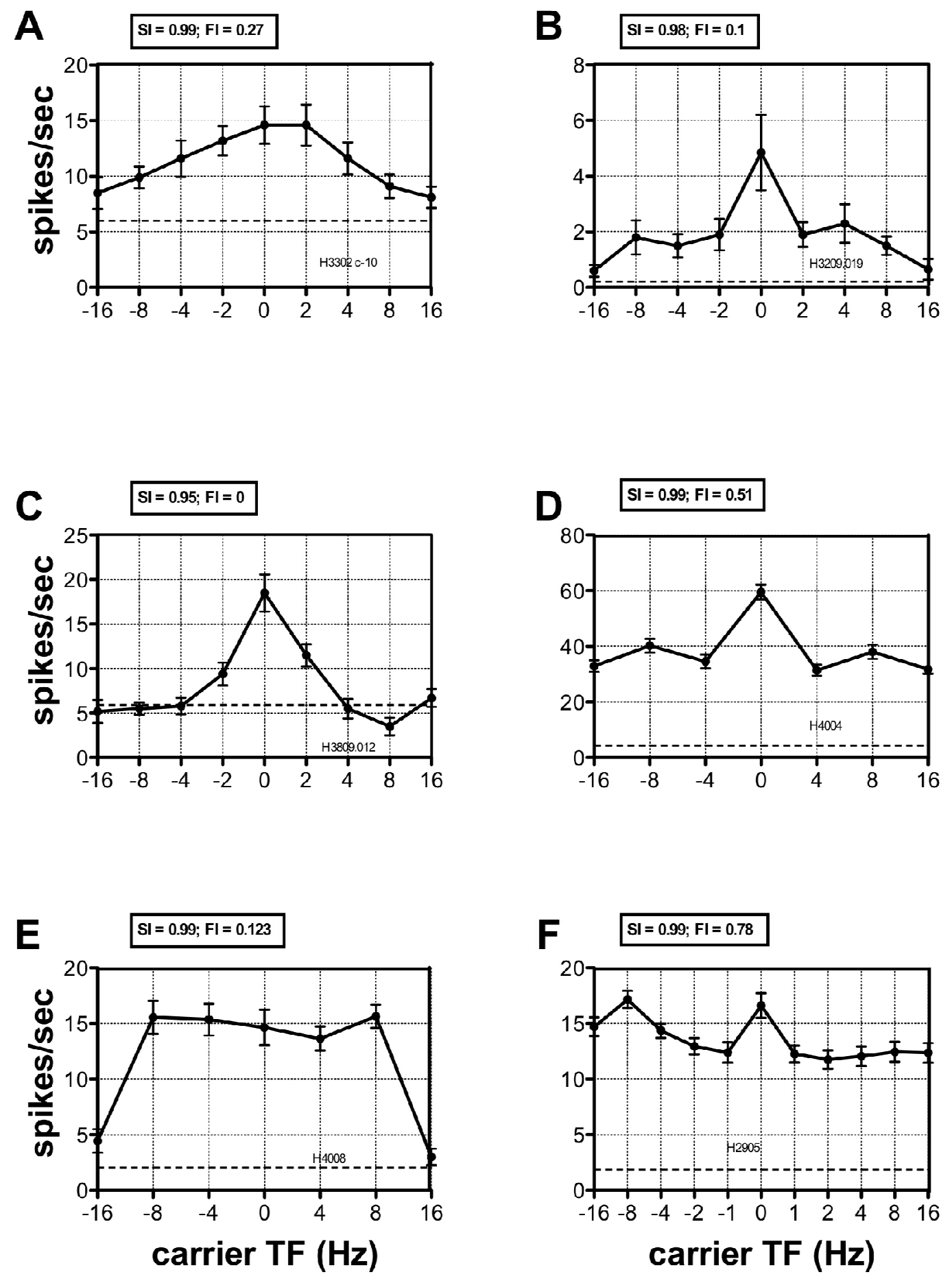
A

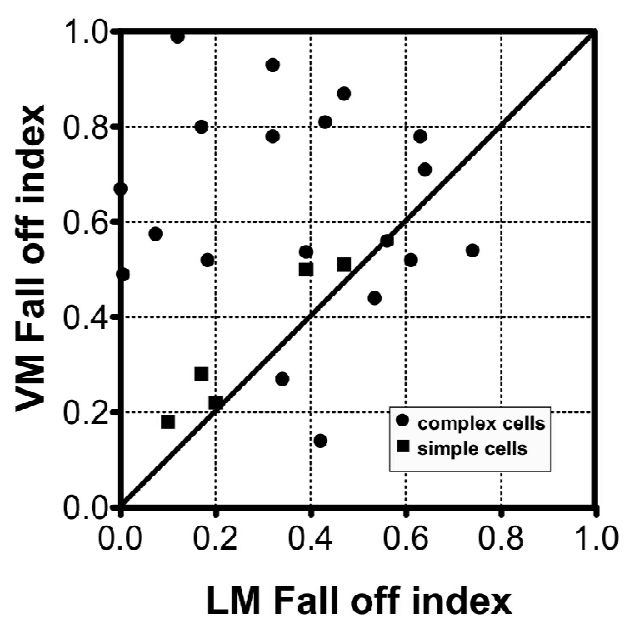

B

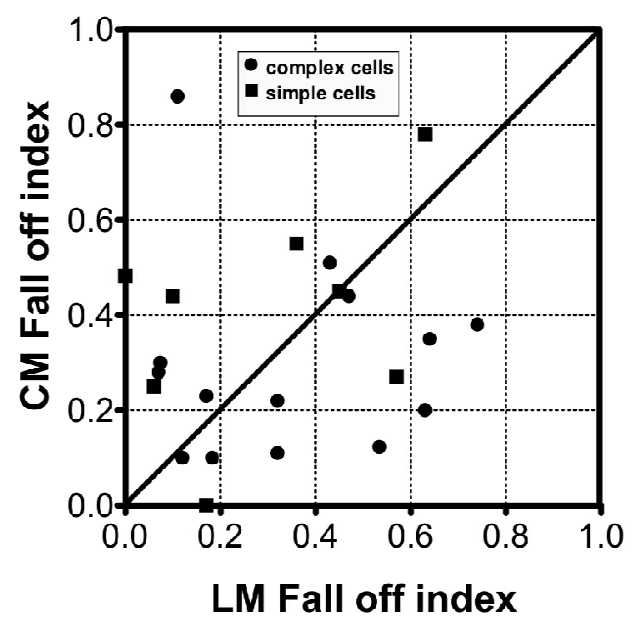

Figure 9.Comparison of fall-off index for "uni-directional" VM and CM gratings with LM gratings. (A) Scatter plot showing neurons' fall-off index for "unidirectional" VM gratings vs. LM gratings for 24 neurons. Most points lie above the equality line (19/24), indicating that for VM gratings neuronal responses fall off relatively less than LM responses at high temporal frequencies. (B) Scatter plot showing neurons' fall-off index for "bi-directional" VM gratings vs. LM gratings for 22 neurons. There was no systematic relationship between fall-off indexes for these two gratings. 
A

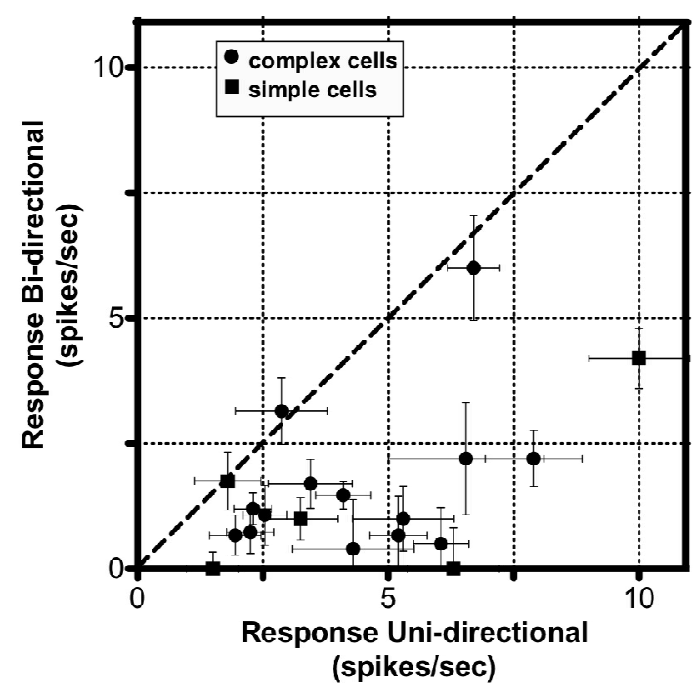

B

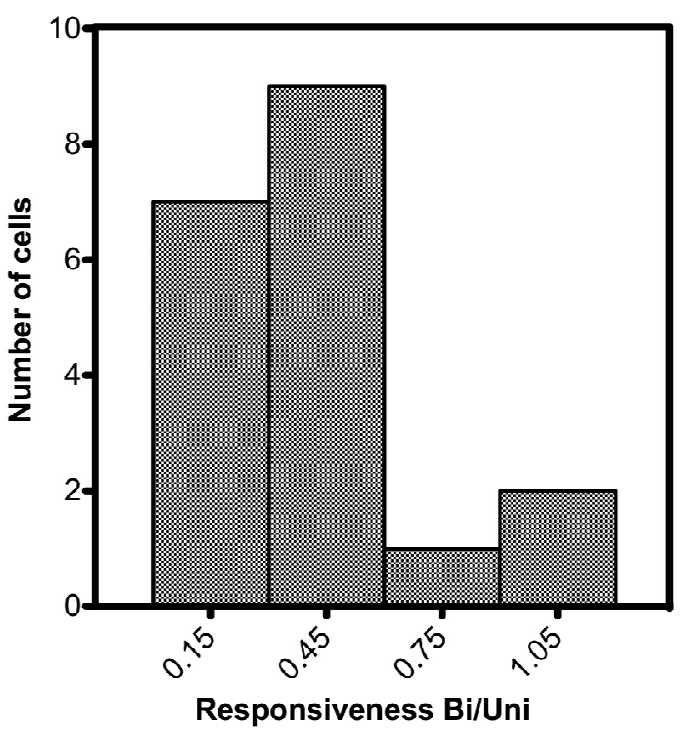

Figure 10. Comparison of peak response amplitudes to "uni-directional" and "bidirectional" VM gratings. (A) Scatter plot showing a neurons' maximum responses (spikes/sec) to "uni-directional" vs. "bi-directional" VM gratings for 19 neurons. Neurons responded more strongly to "uni-directional" compared to "bidirectional" gratings. (B) Histogram showing ratio of response strength to "bidirectional" gratings and "uni-directional" gratings. Ratio of response strength was less than 0.6 for 16 out of 19 neurons (84.2\%). 
Figure 11. Comparison of envelope spatial frequency (SF) tuning to illusory contours (IC), "uni-directional" and "bi-directional" VM gratings and spatial frequency tuning to luminance gratings for four neurons. Neurons preferred the same envelope SFs for "bi-directional" VM gratings and ICs as shown in (A) and (B), while preferred envelope SFs for "uni-directional" VM gratings were higher compared to ICs as shown in (A), (B), (C) and (D). Neurons' preferred SFs for luminance gratings were greater than or equal to preferred envelope SFs for "uni-directional” VM gratings. 

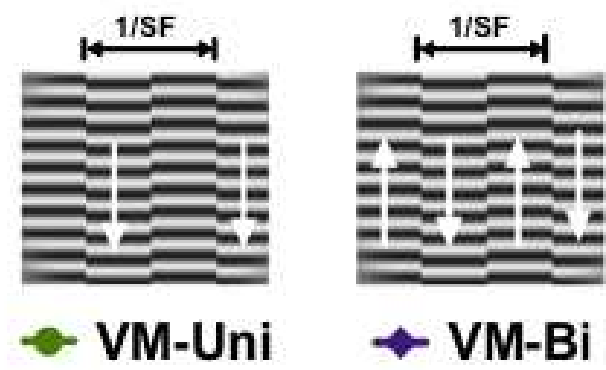

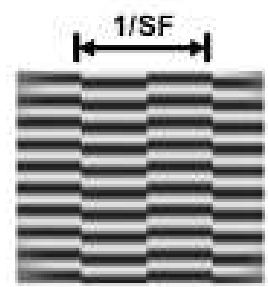

- $\mathrm{CI}$ IC

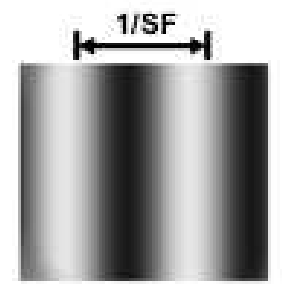

* Lum
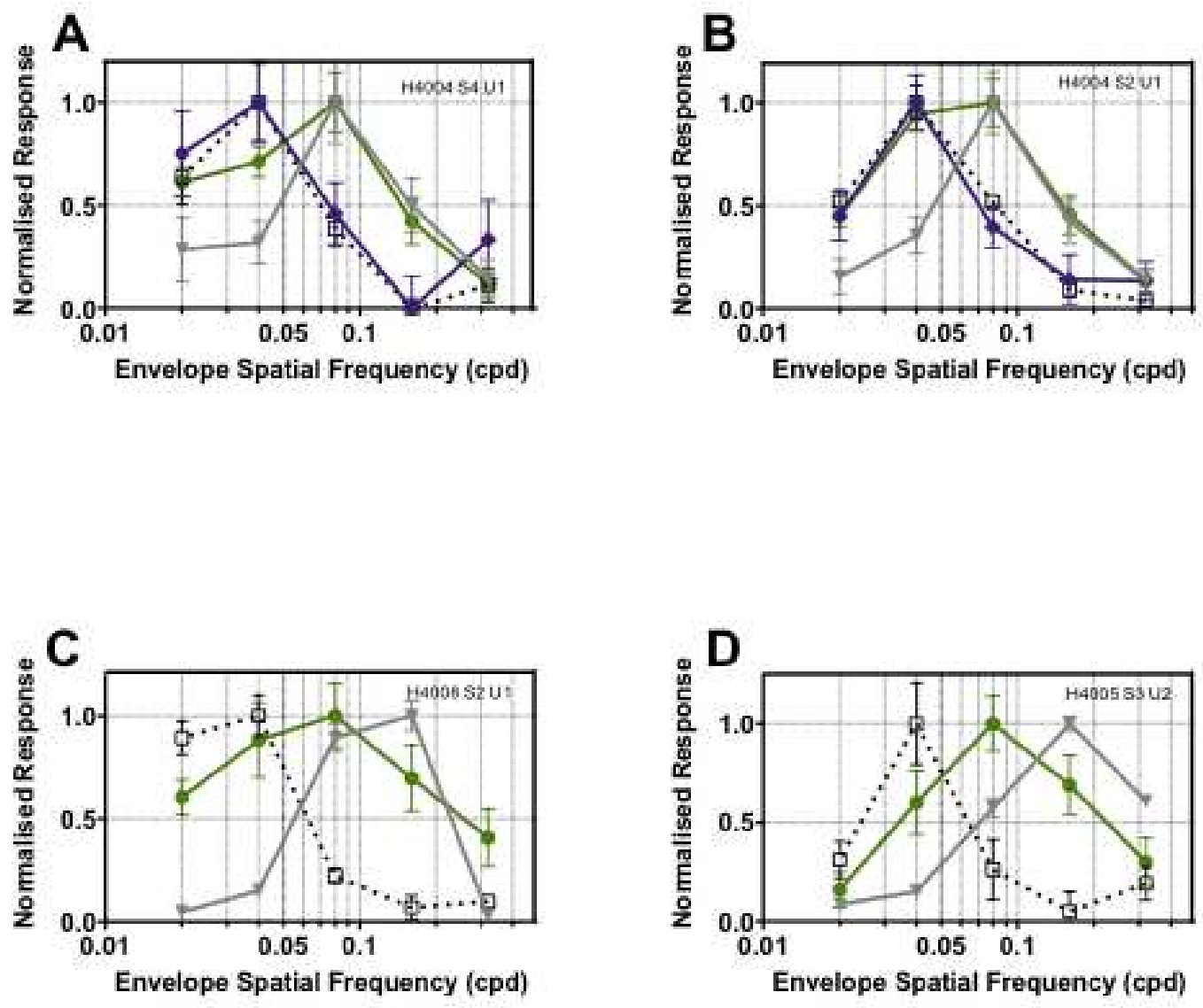
Figure 12. A schematic model accounting for neuronal responses to luminance modulation (LM), contrast modulation (CM) and velocity modulation (VM) gratings. A, A two stream processing scheme, in which first- and second-order responses are mediated by separate, parallel pathways. The top pathway is a coarse spatial scale linear filter (F0), which would be selective to conventional LM gratings. The bottom pathway mediates nonlinear processing of CM and VM gratings. B, A non-linear FRF model that processes CM and VM gratings. First stage of the model consists of early small scale filters (F1) that would be selective for high spatial frequency carrier gratings. Output of these F1 filters are rectified and pooled by late filter F2. This F2 filter would be selective for envelope of CM and VM gratings and would have similar spatio-temporal properties as F0 filter. 
A

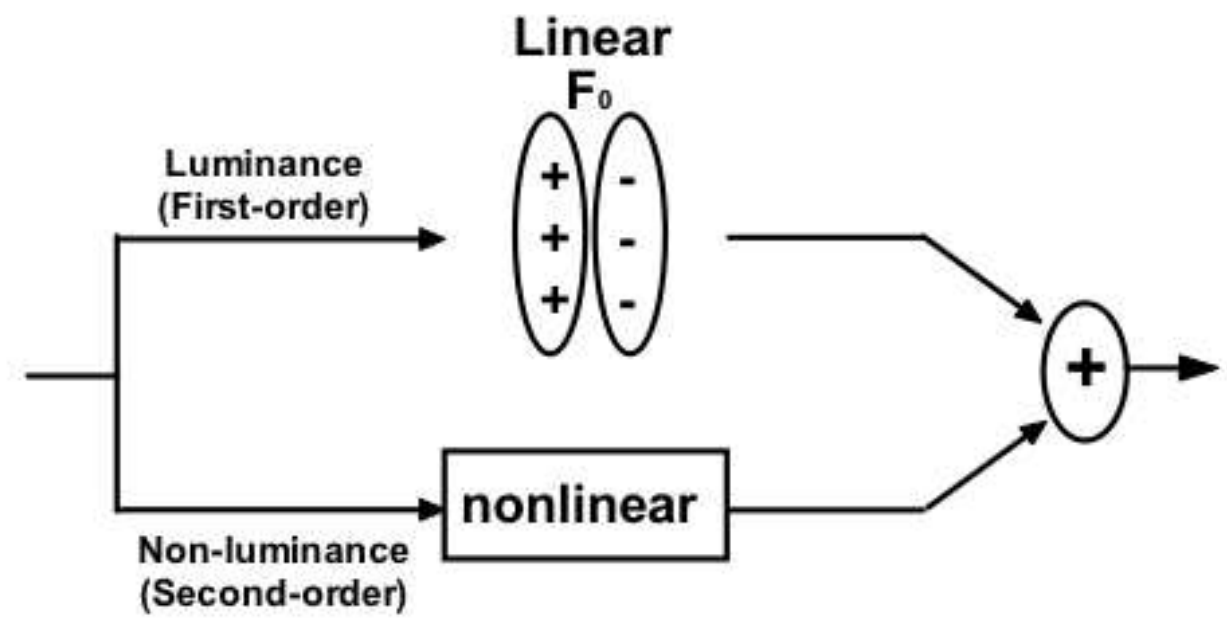

B

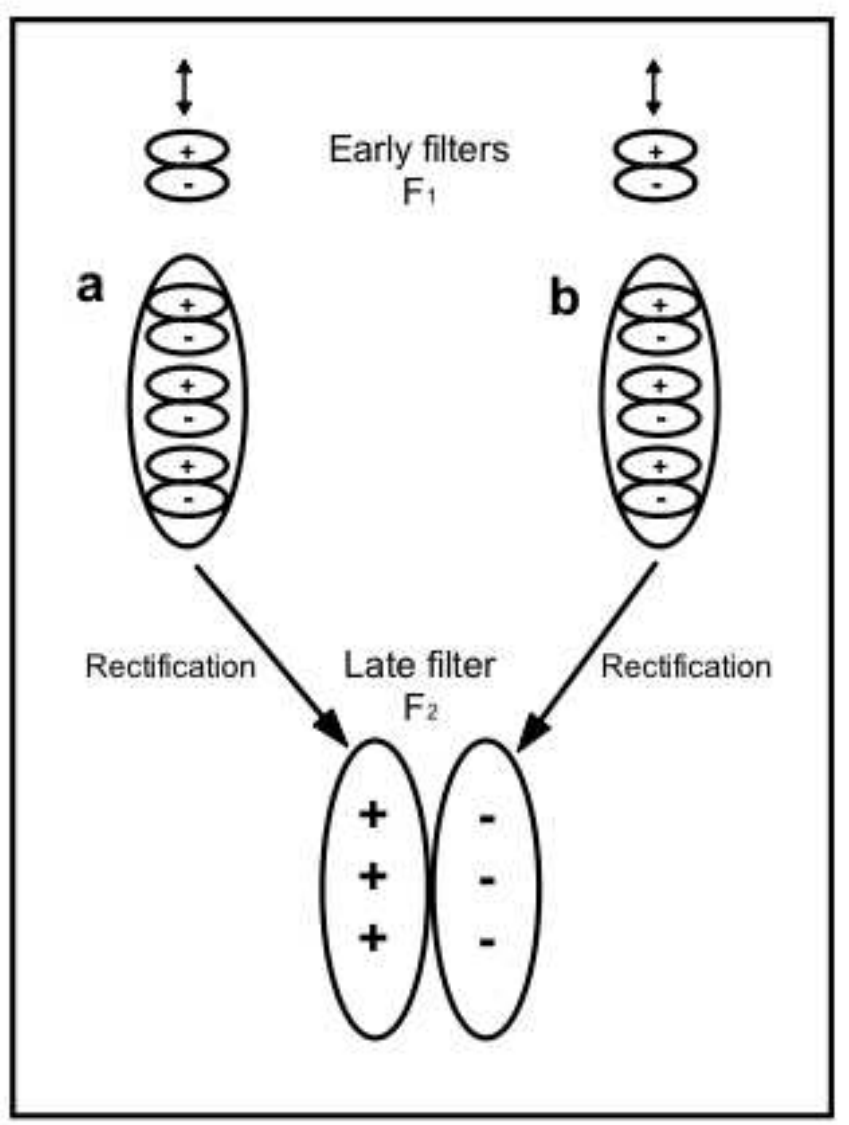

